\title{
STOCHASTIC ACTUARIAL MODELLING OF A DEFINED-BENEFIT SOCIAL SECURITY PENSION SCHEME: AN ANALYTICAL APPROACH
}

\author{
By Subramaniam Iyer
}

\begin{abstract}
Among the systems in place in different countries for the protection of the population against the long-term contingencies of old-age (or retirement), disability and death (or survivorship), defined-benefit social security pension schemes, i.e. social insurance pension schemes, by far predominate, despite the recent trend towards defined-contribution arrangements in social security reforms. Actuarial valuations of these schemes, unlike other branches of insurance, continue to be carried out almost exclusively on traditional, deterministic lines. Stochastic applications in this area, which have been restricted mainly to occasional special studies, have relied on the simulation technique. This paper develops an analytical model for the stochastic actuarial valuation of a social insurance pension scheme. Formulae are developed for the expected values, variances and covariances of and among the benefit expenditure and salary bill projections and their discounted values, allowing for stochastic variation in three key input factors, i.e., mortality, new entrant intake, and interest (net of salary escalation). Each deterministic output of the valuation is thus supplemented with a confidence interval, that is, a range with an attached probability. The treatment covers the premiums under the different possible financial systems for these schemes, which differ from the funding methods of private pensions, as well as the testing of the level of the Fund ratio when the future contributions schedule is pre-determined. Although it is based on a relatively simplified approach and refers only to retirement pensions, with full adjustment in line with salary escalation, the paper brings out the stochastic features of pension scheme projections and illustrates a comprehensive stochastic valuation. It is hoped that the paper will stimulate interest in further research, both of a theoretical and a practical nature, and lead to progressively increasing recourse to stochastic methods in social insurance pension scheme valuations.
\end{abstract}

\section{KEYWORDS}

Social Security; Social Insurance; Pensions; Actuarial Valuation; Deterministic Method; Stochastic Method; Demographic Projections; Financial Projections; Financial Systems

\section{CONTACT ADDRESS}

Subramaniam Iyer, M.Sc., Hon. FIA. 3, Rue Giovanni-Gambini, 1206 Geneva, Switzerland. Tel: (+4122)3469541; E-mail: sniyer_geneva@yahoo.co.uk

\section{INTRODUCTION}

\subsection{Target Area of the Paper}

This paper is concerned with national social security systems, which exist 
in one form or another in almost all countries of the world. These are institutionalised arrangements set up through legislation, with mandatory coverage of specified categories of the population and administered directly by the government or under government control. The stochastic characteristics of private insurance arrangements that supplement or complement the basic protection under the social security system have been discussed extensively in actuarial literature (see, for example, Daykin et al., 1994; Booth et al., 1999) and are not considered in this paper.

An important component of each national social security system is that which covers the long-term contingencies of old-age (or retirement), disability and death (or survivorship). The different approaches to the provision of the corresponding benefits include: (a) social insurance (SI); (b) universal or means-tested schemes (UN/MT); (c) provident funds (PF); and (d) individual retirement accounts (IRA), which include the Notional (or Non-financial) Defined Contribution schemes (NDC) innovated in Sweden and introduced or planned in certain European countries (Holzmann and Palmer, 2003). Occasionally, two or more types may exist simultaneously, particularly during the transitory stage from one approach to another. Categories (a) and (b) are typically defined-benefit (DB) schemes, while (c) and (d) are definedcontribution (DC) arrangements. Table 1, based on information drawn from publications on recent international surveys (Social Security Administration, 2005, 2006a, 2006b, 2007) shows that, despite the trend in recent years from DB to DC in the course of social security reforms (see Daykin and Lewis, 1999; Holzmann and Hinz, 2005), the DB-type social insurance system still predominates in the responding countries.

The same surveys also show that the responding countries span a huge range: in terms of population, extending from as low as 20,000 to as high as 1.3 billion; in terms of per capita GDP, from as low as US\$ 520 to as high as US\$ 40,000. This provides, however, only a very rough indication of the effective population and earnings coverage under social security. While a large proportion of the total or economically active population is covered in the advanced countries, the statutory coverage may relate only to a smaller proportion of the active population in some developing countries. Moreover,

Table 1. Number of countries, by type of long-term social security benefit scheme

$\begin{array}{lccccccr}\text { Area } & \text { SI } & \text { PF } & \text { IRA } & \text { UN/MT } & \text { SI + PF } & \text { SI + IRA } & \text { Total } \\ \text { Africa } & 37 & 3 & 1 & 2 & 1 & - & 44 \\ \text { America } & 25 & - & 1 & - & - & 9 & 35 \\ \text { Asia } & 30 & 11 & - & 4 & 1 & 2 & 48 \\ \text { Europe } & 34 & - & - & - & - & 10 & 44 \\ \text { Total } & 126 & 14 & 2 & 6 & 2 & 21 & 171\end{array}$

Source: Author's analysis of data drawn from issues of "Social Security Programs throughout the World" (US Social Security Administration) 
the effective coverage might fall short of the potential coverage, due to various administrative or other shortcomings (see Gillion et al., 2000).

This paper focuses on social insurance schemes covering the long-term contingencies. These, in principle, are financially autonomous and sustained mainly by member (and, where applicable, employer) contributions, generally related to the individual's insured earnings, which is possibly subject to a threshold and ceiling. They provide pensions for life or until the contingency lasts, typically based on the contributory earnings of the insured person and on the period of contribution. There is usually provision for some, price- or earnings-, indexation of pensions after award. Naturally there are several variations on this basic design, which have been well-documented elsewhere (see, for example, Gillion et al., 2000).

\subsection{Actuarial Valuations of Social Insurance Pension Schemes}

The conduct of actuarial valuations of these schemes, at specified intervals (usually, 3 to 5 years), is often a statutory requirement. In addition, ad hoc valuations may be required, for example, when considering important scheme modifications (Daykin, 2000, 2001). International standards have recently been adopted for such valuations (International Actuarial Association, 2003). The International Labour Office has played a leading role over the years in the development of the mathematical theory and the practice of these valuations (Iyer, 1999; Plamondon et al., 2002).

Being based on the open-fund approach, these schemes may apply one of many possible, partially-funded, financial systems, in the range extending upwards from the unfunded pay-as-you-go system (Iyer, 1999, Chapter 1). The main purpose of the actuarial valuation is to test the long-term solvency of the scheme, that is, to assess if under the existing financing arrangements benefits can be paid and reserve funds maintained at the required levels. On certain occasions, for example when setting up a new scheme or considering important modifications, computations for alternative financial systems will be required (see McGillivray, 1996; Picard, 1996; Daykin, 2000).

The existing actuarial model of the social insurance pension scheme is essentially deterministic (Iyer, 1999). This is in line with the almost universal practice in regard to the actuarial valuation of such schemes (Plamondon et $a l ., 2002)$. Thus, the typical social insurance pension valuation is based on a deterministic scenario, using the "best estimates" of the future values of the determining factors (i.e. rate of population growth, rate of interest, mortality rates etc.). This is often accompanied by two other deterministic valuations, termed "conservative" and "optimistic", purporting to give a range for the valuation results. Additional deterministic variants might be included, to assess the sensitivity of the results to individual key factors. This practice is followed, for example, in the quinquennial valuations of the National Insurance Fund in the United Kingdom (Government Actuary's Department, 2003, 2005). In the case of the Quebec Pension Plan (Regie des Rentes du Quebec, 
2007), the valuation is carried out on a single set of assumptions, subject to sensitivity analysis, taking one factor at a time.

Another example is the annual actuarial valuation of the United States' Old-age and Survivors' and Disability Insurance schemes, where the customary deterministic valuation comprises three scenarios: intermediate, low cost, high cost. However, since 2003, the valuation report has included, in addition, the results from a stochastic model (Social Security Administration, 2004; American Academy of Actuaries, 2005; Board of Trustees of the OASDI, 2007; Buffin, 2007). There are also independent stochastic valuations of the scheme based on other models, i.e. the COBOLT model (Congressional Budget Office, 2001), the TL model (Lee, Anderson and Tuljapurkar, 2003) and the SSASIM model (Burdick and Manchester, 2003).

On the basis of available information, it would appear that the US OASDI is probably the only social insurance pension scheme which carries out a regular, full-fledged stochastic actuarial valuation. However, in the latest valuation of the Canada Pension Plan (Office of the Superintendent of Financial Institutions, Canada, 2007), stochastic methods have been applied for the sensitivity analysis of individual factors. Stochastic methods have also been discussed in the framework of pension reform considerations (Holzmann and Palmer, 2006, Chapter 6). Stochastic applications elsewhere in social security have been generally limited to occasional ad hoc studies, without forming part of an actuarial valuation per se. In this context, it is instructive to refer to the considerable work which has been done by demographers in stochastic human population projections (e.g. Bongaarts and Bulatao (eds), 2000; Keilman et al., 2002; Alho and Spencer, 2005; Booth, 2006) and by ecologists in stochastic animal population dynamics (e.g. Renshaw, 1991; Engen and Saether, 2003).

\subsection{The Case for Stochastic Valuations}

Already more than fifty-five years ago, Frank Redington (Redington, 1952) recognised the probabilistic nature of the factors affecting the future course of an insurance arrangement, and envisioned an "expanding funnel of doubt" in regard to the associated uncertainty. A stochastic valuation, which directly takes this phenomenon into account, is able to provide limits within which an output variable may lie, with attached probabilities, whereas in a deterministic valuation, no probability can be attached to the range between the conservative and optimistic results. Moreover, as different factors act in different directions, the qualification of a specific combination of factors as "conservative" or "optimistic" could be misleading (Lee, 2004). For example, if one such scenario combines high fertility and low mortality and vice versa for the other scenario, these combinations may be unrealistic; while being extreme for one output, e.g. population over normal retirement age, they may not be extreme for another output, say the old-age dependency ratio (Keilman et al., 2002). For this reason, sensitivity analyses in 
deterministic valuations are obliged to be carried out by varying one factor at a time, maintaining the others unchanged, but this does not throw light on the effect of changing factors in combination. This provides another argument for stochastic valuations.

A justification for deterministic valuations could have been the tacit assumption that social security being national in scope, the coverage will generally be large enough to reduce the relative stochastic effect, measured by the coefficient of variation of an output, to insignificance. However it turns out that, unless the covered population is extremely small, the population size has hardly any impact on this coefficient. Thus, the US OASDI, with a coverage amounting to 168 million workers (Board of Trustees, 2007, Table IV.B2., p48), considered it worthwhile to add a stochastic valuation as an adjunct to the deterministic valuation, and the stochastic effects were quite considerable (ibid., Figures VLE1, p162 and VLE2, p163).

Other reasons might be the perception of social insurance pension schemes as unresponsive publicly administered schemes, which do not operate in accordance with generally accepted actuarial principles for the advance funding of future actuarial liabilities under private insurance, and the impression created by the recent emphasis on the macroeconomic effects of social security. However, these factors have not detracted in any way from the justification of (deterministic) valuations of social insurance pension schemes (Daykin, 2000). It is not unreasonable to extend this view to stochastic valuations as well. It should also be appreciated that the financing of social insurance pension schemes is also governed by sound actuarial principles, although somewhat different from those which apply to private pension arrangements (Iyer, 1999).

Some authors have referred to the difficulty of communicating the results of a stochastic valuation to policy makers (Plamondon et al., 2002, p59), who are reputed to be insistent on being provided with a single "best estimate". A parallel view is that a simple methodology — presumably deterministic is to be preferred for these valuations. A diametrically opposite view is that a deterministic projection can be misleading and provides inadequate information for informed policy decisions (Keilman et al., 2002). Clearly there is considerable difference of opinion between experts on the complexity, utility and necessity of stochastic social insurance pension valuations.

\subsection{Application of the Simulation Technique}

As far as is known, all stochastic applications have been based on the simulation methodology. The first step is the projection of the key input variables and their statistical distributions. Thus, in the OASDI valuation, where experience over a long past period is available, this projection used techniques based on auto-regressive (AR) or autoregressive moving average (ARMA) processes (Buffin, 2007). The second step is the drawing of a 
random value for each variable and then simulating the deterministic valuation exercise for this particular choice of inputs. This procedure is repeated several times $(5,000$ times in the case of the OASDI - see Board of Trustees of the OASDI, 2007, Appendix E) and the various results combined to produce a discrete approximation to the (unknown) theoretical distribution of each output variable.

The volume of work involved can be considerable, in view of the number of iterations required. The projection of the input variables and their distributions could be problematical where past experience is limited or unreliable. Moreover, the methodology does not yield the actual distributions of the output variables, but only discrete approximations thereof. This is not meant to detract from the value of the simulation methodology, which remains a practicable approach when dealing with complex stochastic processes. However, it would be instructive, from a theoretical point of view, to have an analytical model, derived from first principles, which would lead to the actual probability distributions of the outputs. As far as is known, such a model has not yet been developed, in view of the inherent mathematical complexities (Plamondon et al., p59).

\subsection{Purpose and Content of this Paper}

The purpose of this paper is to make a first attempt towards the development of such a model. The paper consolidates prior research (Iyer, 2003 and 2006) and revises and extends an earlier model. The model covers retirement pensions only. The principal demographic and financial outputs, both primary and secondary, which are relevant to a valuation, are defined, and formulae are developed for the theoretical means, variances and covariances, of and among these outputs, based on a relatively simplified approach concerning the inputs and the scheme specifications. The theoretical distributions of the outputs are also inferred. A stochastic actuarial valuation of a social insurance pension scheme is demonstrated, as also the effect on the results of alternative assumptions concerning the values of the key inputs or their trends over time.

To preclude undue expectations, it is emphasised that the aim of the paper, principally, is to introduce the concept of a comprehensive stochastic valuation - that is, a valuation which addresses various financial systems and to illustrate the characteristics of such a valuation. This is, in effect, an extension of the existing deterministic model of a social insurance pension scheme (Iyer, 1999). The paper provides theoretical insight into the working out of the stochastic processes underlying the evolution of a pension scheme over time. The paper is not intended to provide a ready-made tool for projecting the inputs and carrying out an actual stochastic valuation. However, the approach developed in the paper could be a starting point for providing an indication of the stochastic variability associated with the retirement pension component of a scheme. 
It is hoped that the paper will contribute to increased awareness, understanding and appreciation of stochastic methods in the social security area, particularly in the context of actuarial valuations and engender further research, both of a theoretical and a practical nature, leading progressively to ever-widening acceptance and application of stochastic methods in social insurance pension valuations.

The paper consists of six sections and four Appendices. The following Section 2 introduces the projection exercise and the stochastic modelling of the inputs. Section 3 presents the primary valuation outputs, i.e. the demographic and financial projections and discounted financial projections. Section 4 discusses the secondary valuation outputs, essentially ratios of primary outputs, such as the demographic ratio or the premium under any of the financial systems discussed in the paper. Section 5 facilitates the numerical illustrations and summarises the results. Section 6 concludes the paper.

Appendix A outlines the pure birth and pure death processes, which provide the theoretical basis for the modelling of the stochastic projections. Appendix B summarises the mathematics of the lognormal distribution, on which the statistical analyses developed in the paper are based. Appendix C provides the background for the statistical treatment of products of variables, and of ratios, such as the secondary outputs. The final Appendix D presents the numerical results of the illustration of the model.

\section{The Projection Exercise: Inputs}

\subsection{Deterministic and Stochastic Projections}

The evolution of a pension scheme is characterised by certain key aggregates, notably the Active Population and the Pensioners and the Insured Salary Bill and the Benefit Expenditure in future years, as well as the latter financial aggregates discounted to the valuation date. These are complex functions of the determining factors, each of which comprises a series of inputs; each input is stochastic, and may be correlated with other inputs of the same factor, or even with the inputs of another factor. The combination of specific values of the inputs is termed a scenario. Evidently, several scenarios are possible, each with an attached probability, constituting the universe of scenarios.

The aim of a projection exercise is to produce values of these primary outputs, based on a particular scenario of factor inputs. A deterministic valuation is essentially the analysis of a limited purposive sample out of the universe of scenarios. A simulation-based stochastic valuation analyses a sizeable random sample of scenarios, and is therefore more scientific and informative. In this paper, it is proposed to derive analytical expressions for 
the expected values, variances and covariances of the key aggregates, based on the probability distribution of all the possible scenarios.

The derivation of analytical formulae for these variances and covariances is extremely complicated and at first sight, seems practically impossible, because of the number of variables involved and the possible correlations between them. However, the problem can be approached by proceeding systematically, in stages, with some approximations and simplification along the way.

The formulae are developed for a simple defined benefit pension scheme with the following characteristics:

- The scheme provides pensions on retirement at a fixed age, denoted by $r$. (Other benefits, such as disability or survivors' pensions, are not considered.)

- All members enter the scheme at a fixed age, denoted by $b$.

- The pension is a function of the service period and the final salary of the member, and is fully indexed to salary escalation after retirement.

\subsection{Methodologies for Deterministic Projections}

Before proceeding to consider stochastic projections, it would be useful to review briefly the practical methodologies for deterministic projections. The following summary excludes several details and refinements; for a comprehensive treatment, see Iyer, 1999, Chapter 5 and Plamondon et al., 2002, Chapters 9 and 10.

One possible approach, when the scheme covers a large proportion of the population, is to project the national population first and to derive the scheme related projections by applying appropriate proportions to the national population projections.

The other, more general, approach is to establish independent projections for the social insurance pension scheme. This approach is described below, in broad outline.

The starting point is the sub-division of the initial active population and each category of pensioner, and the assumed future entrants, by sex, and by age within each sex. This allows the application of the cohort component method, where each age-related cohort is projected forward, using a single or multiple decrement table, as appropriate, and the results for different cohorts combined to produce time-related aggregates.

For the active population projections, a multiple decrement table is likely to be used, allowing for at least a disability decrement, in addition to the mortality decrement. The national life tables provide a starting point for the mortality basis. The mortality level of the active population is presumed to be lower than that of the general population, implying a correspondingly higher mortality level for the disabled population, the gap between the two reducing with increasing age. In practice, starting from an assumed mortality level for the disabled population, the active service mortality may be 
determined consequentially. Disability incidence rates are differentiated by sex and are understood to increase with age.

The financial projections are obtained by applying appropriate factors to each component of the demographic projections, i.e. for active persons, the corresponding projected average insured earnings and, for pensioners, the projected average pension, including any indexation adjustments after award.

The development in this paper is on the lines of the scheme-specific projection approach. Although only retirement pensions are considered, the disability decrement is taken into account in the formulae for the active population projections.

\subsection{The Underlying Stochastic Processes}

Given the initial (active and retired) population and the salaries and pensions in force at valuation date, the primary outputs can be regarded as the outcome of one or more of several individual processes. A pension scheme with pension adjustment in line with salary escalation can be valued by allowing for salary escalation indirectly, through a net interest factor. Thus, the key processes relevant to the evolution of the scheme are:

- The new entrant growth process

- The survival process (for active and retired lives)

- The discount process, at interest net of salary escalation.

The demographic projections and financial projections (ignoring salary escalation) are the outcome of the first two processes, and the discounted financial projections the outcome of the three processes.

The new entrant growth process affecting the evolution of a national pension scheme is, by nature, both demographic and economic. On the one hand, the supply depends on past and future births while the demand is conditioned by current and projected economic growth and employment. Where a large proportion of the population is covered under social insurance, the future entrants would be closely linked to national demographics. Where the proportion of the population covered is small, the link with the economic factors affecting the covered sectors would be predominant. In this paper, however, the new entrant growth process is treated as an autonomous process. This is a simplification.

Over the age range $(b, r)$, the survival process for active persons includes, in addition to mortality, other causes of exit from the active insured status, i.e. disability in particular.

The classical approach has been to regard these processes as deterministic, leading to deterministic aggregates and on to deterministic valuations. However, in fact, these various processes are stochastic and possibly intercorrelated.

Strictly speaking, the new entrant and the survival processes are "integral" processes - see Appendix A — since the outcome at each stage has to be whole 
numbers of persons, whereas this restriction need not apply to the discount process, as fractions of the monetary unit are admissible. Thus, the variances and covariances of the primary outputs have two components: a "demographic" component which arises because of differences between individuals in the outcome at each stage of the integral processes, and an "environmental" component, which is the true stochasticity effect (see Engen et al., 1998). However, at the level of the populations covered under social insurance pension schemes, the "demographic" component is relatively minor, and can be ignored. This amounts to regarding the new entrant and survival processes also as "fractional" processes. This simplifies the expressions and facilitates the statistical analysis. However, the full expressions, including the demographic component, are derived below.

The formulae developed below allow for stochasticity of the disability element of the survival process to be taken into account, but subsequently (including the numerical illustrations) this element is treated as deterministic.

\subsection{Definitions and Notation}

For the purposes of this paper, the stochastic processes are characterised by the following instantaneous rates of change:

- The force of growth of the number of new entrants, $\rho(t)$ at time $t$.

- The force of population decrement, $\mu(x, t)$ at age $x$ and time $t$. $\mu(x, t)=\mu^{(1)}(x, t)+\mu^{(2)}(x, t)$, where $\mu^{(1)}(x, t)$ refers to mortality and $\mu^{(2)}(x, t)$ to disability $\left(\mu^{(2)}(x, t)=0\right.$ for $\left.x \geq r\right)$.

- The force of interest (net of salary escalation), $\delta(t)$ at time $t$.

Each stochastic force is assumed to be normally distributed, with attendant expected values, variances and intra-factor covariances. The force of mortality is assumed to be correlated both age-wise and time-wise. The stochastic factors are assumed to be mutually independent. It follows that all integrals of the forces are normally distributed, by analogy with the distribution of a sum of normal variables.

Expected values, variances and covariances of stochastic functions such as $f(\cdot), g(\cdot)$, are denoted, respectively, by $E(f), V(f)$, and $\operatorname{Cov}(f, g)$. A special notation - see 2.4.1 — indicates the exponential function of the integrals of the forces, as well as of the expected values, variances and covariances of the integrals. All integrals are taken over the time variable.

A pre-superscript $(\rho, \mu, \delta)$ will identify the factor of reference (growth, population decrement, or interest). In the case of population decrement, an additional pre-superscript in brackets will identify the cohort, i.e. indicate when the cohort attained age $b$. The post-, sub- and superscripts will indicate the time range(s) of the integral.

Equation numbers in the body of the paper are indicated as $(\cdot)$, in the Appendices as [.]. The key equations for the valuation are identified by the sign (*). 
2.4.1 The new entrant growth factor and the interest factor

The exponential function of the integral of the force of growth is indicated as follows and defined as shown:

$$
{ }^{\rho} \boldsymbol{I}_{h}^{k}=\exp \left(\int_{h}^{k} \rho(z) d z\right)
$$

The relevant functions of the expected value, variance and covariance are indicated and defined as follows:

$$
\left.\begin{array}{c}
{ }^{\rho} e_{h}^{k}=\exp \left(E\left({ }^{\rho} I_{h}^{k}\right)\right)=\exp \left(\int_{h}^{k} E(\rho(z)) d z\right) \\
{ }^{\rho} v_{h, h}^{k, k}=\exp \left(V\left({ }^{\rho} I_{h}^{k}\right)\right)=\exp \left(\int_{h}^{k} \int_{h}^{k} \operatorname{Cov}(\rho(z), \rho(w)) d w d z\right) \\
{ }^{\rho} v_{h, h^{*}}^{k, k^{*}}=\exp \left(\operatorname{Cov}\left({ }^{\rho} I_{h}^{k},{ }^{\rho} I_{h^{*}}^{k^{*}}\right)\right)=\exp \left(\int_{h}^{k} \int_{h^{*}}^{k^{*}} \operatorname{Cov}(\rho(z), \rho(w)) d w d z\right) .
\end{array}\right\}
$$

The expression for $\operatorname{Cov}\left({ }^{\rho} I_{h}^{k},{ }^{\rho} I_{h^{*}}^{k^{*}}\right)$, and by extension for $V\left({ }^{\rho} I_{h}^{k}\right)$ is the continuous-time analogue of the formula for the covariance between two linear functions of correlated variables — see, for example, Parker, 1994, p170 (19). A useful relationship is:

$$
{ }^{\rho} v_{h, h^{*}}^{k, k^{*}}=\left({ }^{\rho} v_{0,0}^{k, k^{*}}\right)\left({ }^{\rho} v_{0,0}^{h, k^{*}}\right)^{-1}\left({ }^{\rho} v_{0,0}^{k, h^{*}}\right)^{-1}\left({ }^{\rho} v_{0,0}^{h, h^{*}}\right) .
$$

The case of the interest factor is similar, except that $\delta$ will replace $\rho$.

\subsubsection{The population decrement factor}

The survival process is slightly more complex because age and time need to be taken unto account. It should be noted that when the cohort is identified by $(j)$ the age of the cohort at time $z$ is $b-j+z$. 


$$
\begin{aligned}
{ }^{\mu(j)} e_{h}^{k} & =\exp \left(E\left({ }^{\mu(j)} I_{h}^{k}\right)\right)=\exp \left(\int_{h}^{k} E(\mu(b-j+z, z)) d z\right) \\
{ }^{\mu(j)} v_{h, h}^{k, k} & =\exp \left(V\left({ }^{\mu(j)} I_{h}^{k}\right)\right) \\
& =\exp \left(\int_{h}^{k} \int_{h}^{k} \operatorname{Cov}(\mu(b-j+z, z), \mu(b-j+w, w)) d w d z\right) \\
\mu(j) v_{h, h^{*}}^{k, k^{*}} & =\exp \left(\operatorname{Cov}\left({ }^{\mu(j)} I_{h}^{k},{ }^{\mu(j)} I_{h^{*}}^{k^{*}}\right)\right) \\
& =\exp \left(\int_{h}^{k} \int_{h^{*}}^{k^{*}} \operatorname{Cov}(\mu(b-j+z, z), \mu(b-j+w, w)) d w d z\right) \\
\mu\left(j, j^{*}\right) v_{h, h^{*}}^{k, k^{*}} & =\exp \left(\operatorname{Cov}\left({ }^{\mu(j)} I_{h}^{k},{ }^{\mu\left(j^{*}\right)} I_{h^{*}}^{k^{*}}\right)\right) \\
& =\exp \left(\int_{h}^{k} \int_{h^{*}}^{k^{*}} \operatorname{Cov}\left(\mu(b-j+z, z), \mu\left(b-j^{*}+w, w\right)\right) d w d z\right) .
\end{aligned}
$$

Each covariance term in (4) can be further developed. For example,

$$
\begin{aligned}
\operatorname{Cov}( & \left.\mu(b-j+z, z), \mu\left(b-j^{*}+w, w\right)\right) \\
= & \operatorname{Cov}\left(\mu^{(1)}(b-j+z, z), \mu^{(1)}\left(b-j^{*}+w, w\right)\right) \\
& +\operatorname{Cov}\left(\mu^{(1)}(b-j+z, z), \mu^{(2)}\left(b-j^{*}+w, w\right)\right) \\
& +\operatorname{Cov}\left(\mu^{(2)}(b-j+z, z), \mu^{(1)}\left(b-j^{*}+w, w\right)\right) \\
& +\operatorname{Cov}\left(\mu^{(2)}(b-j+z, z), \mu^{(2)}\left(b-j^{*}+w, w\right)\right) .
\end{aligned}
$$

If $\mu^{(2)}(x, t)$ is deterministic, the RHS in the above will reduce to the first term.

\section{The Valuation: Primary Outputs}

\subsection{Cohort-component Projection Formulae}

The population is assumed to be a continuous function of age $x$ and time $t$, and is denoted by $P(x, t), b \leq x<\omega, b$ being the entry age and $\omega$ the limit of life. The population in the age interval $(x, x+d x)$ at time $t$ is therefore $P(x, t) d x$. The symbol $P_{(x)}^{(t)}$ is also used for $P(x, t)$. Moreover, the symbols ${ }^{i} P_{(x)}^{(t)}$ and ${ }^{n} P_{(x)}^{(t)}$ are used to distinguish between those surviving out of the population on valuation date (initial population) and out of subsequent entrants (new entrants). The starting population, $P(x, 0)$ is assumed to be given. The number of new entrants in the time interval $(t, t+d t)$ is $P(b, t) d t$.

Using the properties of the lognormal distribution, and with reference to Appendix A, the following formulae are derived. 


\subsubsection{Initial population cohorts}

${ }^{i} P_{(x)}^{(t)}$ is generated from ${ }^{i} P_{(x-t)}^{(0)}$ by a survival process, assumed to be a stochastic integral process. With reference to formulae [8] and [15] of Appendix A, the expected value and variance of ${ }^{i} P_{(x)}^{(t)}$ are given by the formulae $(j=t-x+b)$,

$$
\left.\begin{array}{rl}
E\left({ }^{i} P_{(x)}^{(t)}\right) & ={ }^{i} P_{(x-t)}^{(0)}\left({ }^{\mu(j)} e_{0}^{t}\right)^{-1}\left({ }^{\mu(j)} v_{0,0}^{t, t}\right)^{1 / 2} \\
V\left({ }^{i} P_{(x)}^{(t)}\right) & =\left(E\left({ }^{i} P_{(x)}^{(t)}\right)\right)\left(\left|1-\left({ }^{\mu(j)} e_{0}^{t}\right)^{-1}\left({ }^{\mu(j)} v_{0,0}^{t, t}\right)^{3 / 2}\right|\right)+\left(E\left({ }^{i} P_{(x)}^{(t)}\right)\right)^{2}\left(\left({ }^{\mu(j)} v_{0,0}^{t, t}\right)-1\right) .
\end{array}\right\}
$$

With reference to formulae [15] and [21] of Appendix A, the covariance between ${ }^{i} P_{(x)}^{(t)}$ and ${ }^{i} P_{(y)}^{(u)}$, (a) when they are from the same cohort $(x>y, t>u)$ or (b) from different cohorts, is respectively $(j=t-x+b$, $\left.j^{*}=u-y+b\right)$,

$$
\left.\begin{array}{rl}
(a) \operatorname{Cov}\left\{{ }^{i} P_{(x)}^{(t)},{ }^{i} P_{(y)}^{(u)}\right\}= & \left(E\left({ }^{i} P_{(x)}^{(t)}\right)\right)\left(\left|1-\left({ }^{\mu(j)} e_{0}^{u}\right)^{-1}\left({ }^{\mu(j)} v_{0,0}^{u, u}\right)^{1 / 2}\left({ }^{\mu(j)} v_{0,0}^{t, u}\right)\right|\right) \\
& +\left(E\left({ }^{i} P_{(x)}^{(t)}\right)\right)\left(E\left({ }^{i} P_{(y)}^{(u)}\right)\right)\left(\left(^{\mu(j)} v_{0,0}^{t, u}\right)-1\right) \\
(b) \operatorname{Cov}\left\{{ }^{i} P_{(x)}^{(t)},{ }^{i} P_{(y)}^{(u)}\right\}= & \left.\left(E\left({ }^{i} P_{(x)}^{(t)}\right)\right)\left(E\left({ }^{i} P_{(y)}^{(u)}\right)\right)\left({ }^{\mu\left(j, j^{*}\right)} v_{0,0}^{t, u}\right)-1\right) .
\end{array}\right\}
$$

\subsubsection{New entrant cohorts}

${ }^{n} P_{(x)}^{(t)}$ is generated by two successive independent stochastic integral processes, the new entrant growth process over $(0, j)$ and the survival process over $(j, t)$, starting from $P(b, 0)$ where $j=t-x+b$. This gives, with reference to formulae [17] and [18] of Appendix A,

$$
\left.\begin{array}{rl}
E\left({ }^{n} P_{(x)}^{(t)}\right)= & P_{(b)}^{(0)}\left({ }^{\rho} e_{0}^{j}\right)\left({ }^{\mu(j)} e_{j}^{t}\right)^{-1}\left({ }^{\rho} v_{0,0}^{j, j}\right)^{1 / 2}\left({ }^{\mu(j)} v_{j, j}^{t, t}\right)^{1 / 2} \\
V\left({ }^{n} P_{(x)}^{(t)}\right)= & \left(E\left({ }^{n} P_{(x)}^{(t)}\right)\right)\left(\begin{array}{c}
\left|1-\left({ }^{\mu(j)} e_{j}^{t}\right)^{-1}\left({ }^{\mu(j)} v_{j, j}^{t, t}\right)^{3 / 2}\right| \\
+\left({ }^{\mu(j)} e_{j}^{t}\right)^{-1}\left({ }^{\mu(j)} v_{j, j}^{t, t}\right)^{3 / 2}\left|\left({ }^{\rho} e_{0}^{j}\right)\left({ }^{\rho} v_{0,0}^{j, j}\right)^{3 / 2}-1\right|
\end{array}\right) \\
& +\left(E\left({ }^{n} P_{(x)}^{(t)}\right)\right)^{2}\left(\left({ }^{\rho} v_{0,0}^{j, j}\right)\left({ }^{\mu(j)} v_{j, j}^{t, t}\right)-1\right) .
\end{array}\right\}
$$

If ${ }^{n} P_{(x)}^{(t)}$ and ${ }^{n} P_{(y)}^{(u)}$ are from the same cohort $(x>y, t>u)$, with reference to formula [19] of Appendix A, their covariance is given by,

$\operatorname{Cov}\left({ }^{n} P_{(x)}^{(t)},{ }^{n} P_{(y)}^{(u)}\right)$

$$
\left.\begin{array}{rl}
= & \left(E\left({ }^{n} P_{(x)}^{(t)}\right)\right)\left(\begin{array}{c}
\left|1-\left({ }^{\mu(j)} e_{j}^{u}\right)^{-1}\left({ }^{\mu(j)} v_{j, j}^{u, u}\right)^{1 / 2}\left({ }^{\mu(j)} v_{j, j}^{t, u}\right)\right| \\
+\left({ }^{\mu(j)} e_{j}^{u}\right)^{-1}\left({ }^{\mu(j)} v_{j, j}^{u, u}\right)^{1 / 2}\left({ }^{\mu(j)} v_{j, j}^{t, u}\right)\left|\left({ }^{\rho} e_{0}^{j}\right)\left({ }^{\rho} v_{0,0}^{j, j}\right)^{3 / 2}-1\right|
\end{array}\right) \\
& +\left(E\left({ }^{n} P_{(x)}^{(t)}\right)\right)\left(E\left({ }^{n} P_{(y)}^{(u)}\right)\right)\left(\left({ }^{\rho} v_{0,0}^{j, j}\right)\left({ }^{\mu(j)} v_{j, j}^{t, u}\right)-1\right) .
\end{array}\right\}
$$


If ${ }^{n} P_{(x)}^{(t)}$ and ${ }^{n} P_{(y)}^{(u)}$ are from different cohorts $\left(j=t-x+b, j^{*}=u-y+b\right.$, $j^{*}<j$ ), with reference to formulae [21] of Appendix A,

$$
\begin{aligned}
& \operatorname{Cov}\left({ }^{n} P_{(x)}^{(t)},{ }^{n} P_{(y)}^{(u)}\right) \\
& =\left(E\left({ }^{n} P_{(x)}^{(t)}\right)\right)\left(\left({ }^{\mu\left(j^{*}\right)} e_{j^{*}}^{u}\right)^{-1}\left({ }^{\mu\left(j^{*}\right)} v_{j^{*}, j^{*}}^{u, u}\right)^{1 / 2}\left({ }^{\mu\left(j, j^{*}\right)} v_{j, j^{*}}^{t, u}\right)\left|\left({ }^{\rho} e_{0}^{j^{*}}\right)\left({ }^{\rho} v_{0,0}^{j^{*}, j^{*}}\right)^{1 / 2}\left({ }^{\rho} v_{0,0}^{j, j^{*}}\right)-1\right|\right) \\
& \quad+\left(E\left({ }^{n} P_{(x)}^{(t)}\right)\right)\left(E\left({ }^{n} P_{(y)}^{(u)}\right)\right)\left(\left({ }^{\rho} v_{0,0}^{j, j^{*}}\right)\left({ }^{\mu\left(j, j^{*}\right)} v_{j, j^{*}}^{t, u}\right)-1\right) .
\end{aligned}
$$

Finally, if ${ }^{n} P_{(x)}^{(t)}$ is from a new entrant cohort and ${ }^{i} P_{(y)}^{(u)}$ is from an initial population cohort, with reference to formulae [21] of Appendix A,

$$
\operatorname{Cov}\left({ }^{n} P_{(x)}^{(t)},{ }^{n} P_{(y)}^{(u)}\right)=\left(E\left({ }^{n} P_{(x)}^{(t)}\right)\right)\left(E\left({ }^{n} P_{(y)}^{(u)}\right)\right)\left(\left({ }^{\mu\left(j, j^{*}\right)} v_{j, 0}^{t, u}\right)-1\right) .
$$

\subsection{Demographic Projection Aggregates}

Denoting by $A(t)$ the total Active Population at time $t$ and by $R(t)$ the total Retired Population, the expected values and covariances of these functions can be expressed as,

$$
\left.\begin{array}{rl}
E(A(t)) & =\int_{b}^{r} E(P(x, t)) d x \\
E(R(t)) & =\int_{r}^{\sigma} E(P(x, t)) d x \\
\operatorname{Cov}\{A(t), A(u)\} & =\int_{b}^{r} \int_{b}^{r} \operatorname{Cov}\{P(x, t), P(y, u)\} d y d x \\
\operatorname{Cov}\{R(t), R(u)\} & =\int_{r}^{\sigma} \int_{r}^{\sigma} \operatorname{Cov}\{P(x, t), P(y, u)\} d y d x \\
\operatorname{Cov}\{R(t), A(u)\} & =\int_{r}^{\infty} \int_{b}^{r} \operatorname{Cov}\{P(x, t), P(y, u)\} d y d x .
\end{array}\right\}
$$

In particular, by putting $u=t$ in the last three expressions, expressions for $V(A(t)), V(R(t)), \operatorname{Cov}\{R(t), A(t)\}$ can be obtained. The expressions can be further developed by distinguishing between initial population and new entrant cohorts. Let $b(t)$ denote the upper age limit of the active new entrant population and $r(t)$ the upper age limit of the retired new entrant population. Then, $b(t)$ is defined for $t<r-b$ as $b(t)=b+t$ with $b(t)=r$ for $t>r-b$. $r(t)$ is defined for $t>r-b$ as $r(t)=b+t$ with $r(t)=r$ for $t<r-b$ and $r(t)=\omega$ for $t>\omega-b$. With the understanding that an integral has zero value when the upper and lower limits are equal, the first two and the last of the expressions in (11) can be expanded, in terms of the results developed in Section 3.1 above, as, 


$$
\begin{aligned}
E(A(t))= & \int_{b}^{b(t)} E\left({ }^{n} P_{(x)}^{(t)}\right) d x+\int_{b(t)}^{r} E\left({ }^{i} P_{(x)}^{(t)}\right) d x \\
E(R(t))= & \int_{r}^{r(t)} E\left({ }^{n} P_{(x)}^{(t)}\right) d x+\int_{r(t)}^{\sigma} E\left({ }^{i} P_{(x)}^{(t)}\right) d x \\
\operatorname{Cov}\{R(t), A(u)\}= & \int_{r}^{r(t)} \int_{b}^{b(u)} \operatorname{Cov}\left\{{ }^{n} P_{(x)}^{(t)},{ }^{n} P_{(y)}^{(u)}\right\} d y d x \\
& +\int_{r}^{r(t)} \int_{b(u)}^{r} \operatorname{Cov}\left\{{ }^{n} P_{(x)}^{(t)},{ }^{i} P_{(y)}^{(u)}\right\} d y d x \\
& +\int_{r(t)}^{\omega} \int_{b}^{b(u)} \operatorname{Cov}\left\{P_{(x)}^{i},{ }^{(t)} P_{(y)}^{(u)}\right\} d y d x \\
+ & \int_{r(t)}^{\omega} \int_{b(u)}^{r} \operatorname{Cov}\left\{{ }^{i} P_{(x)}^{(t)},{ }^{i} P_{(y)}^{(u)}\right\} d y d x .
\end{aligned}
$$

The other expressions can be developed on similar lines.

\subsection{Financial Projection Aggregates, Ignoring Salary Escalation}

It is assumed that the growth of an active member's salary is governed apart from salary escalation due to the increase in the general level of earnings - by the progression due to age/seniority along a salary scale $s(x)$, $b \leq x \leq r$, assumed deterministically. Moreover, pensions are assumed to be fully indexed to salary escalation after award.

Average annual salaries and average annual pensions at the outset are assumed to be given and are denoted by $s a(x, 0), p e(x, 0)$. The pension rate for the initial active population aged $x$ is denoted by $\operatorname{pr}(x), \operatorname{pr}(b)$ being the pension rate for new entrants. The population element $P(x, t)$ will have an associated financial factor - ignoring salary escalation — as follows (the pre-superscript indicates whether the population element is currently active (a) or retired $(r)$, and when necessary to make a distinction, if it belongs to an initial population cohort $(i)$ or to a new entrant cohort $(n)$ ):

For an active element deriving from an initial population cohort,

$$
{ }^{i a} f_{(x)}^{(t)}=s a(x-t, 0) \frac{s(x)}{s(x-t)} .
$$

For a retired element deriving from an initial active population cohort,

$$
{ }^{i r} f_{(x)}^{(t)}=s a(x-t, 0) \frac{s(r)}{s(x-t)} \operatorname{pr}(x-t) .
$$


For a retired element deriving from an initial retired population cohort,

$$
{ }^{i r} f_{(x)}^{(t)}=p e(x-t, 0)
$$

For an active element deriving from a new entrant cohort,

$$
{ }^{n a} f_{(x)}^{(t)}=s a(b, 0) \frac{s(x)}{s(b)}
$$

For a retired element deriving from a new entrant cohort,

$$
{ }^{n r} f_{(x)}^{(t)}=s a(b, 0) \frac{s(r)}{s(b)} \operatorname{pr}(b) .
$$

Denoting the total salary bill function by $S(t)$ and the total benefit function by $B(t)$ - both, ignoring salary escalation - the formulae for their expected values, variances and covariances can be derived from the formulae for the demographic projections by applying the relevant financial factors. For example, the financial projection formulae corresponding to (12) are,

$$
\left.\begin{array}{rl}
E(S(t))= & \int_{b}^{b(t)}{ }^{n a} f_{(x)}^{(t)} E\left({ }^{n} P_{(x)}^{(t)}\right) d x+\int_{b(t)}^{r}{ }^{i a} f_{(x)}^{(t)} E\left({ }^{i} P_{(x)}^{(t)}\right) d x \\
E(B(t))= & \int_{r}^{r(t)}{ }^{n r} f_{(x)}^{(t)} E\left({ }^{n} P_{(x)}^{(t)}\right) d x+\int_{r(t)}{ }^{i r} f_{(x)}^{(t)} E\left({ }^{i} P_{(x)}^{(t)}\right) d x \\
\operatorname{Cov}\{B(t), S(u)\}= & \int_{r}^{r(t)}{ }^{n r} f_{(x)}^{(t)} \int_{b}^{b(u)}{ }^{n a} f_{(y)}^{(u)} \operatorname{Cov}\left\{{ }^{n} P_{(x)}^{(t)},{ }^{n} P_{(y)}^{(u)}\right\} d y d x \\
& +\int_{r}^{r(t)}{ }^{n r} f_{(x)}^{(t)} \int_{b(u)}^{r}{ }^{i a} f_{(y)}^{(u)} \operatorname{Cov}\left\{{ }^{n} P_{(x)}^{(t)},{ }^{i} P_{(y)}^{(u)}\right\} d y d x \\
& +\int_{r(t)}{ }^{i r} f_{(x)}^{(t)} \int_{b}^{b(u)}{ }^{n a} f_{(y)}^{(u)} \operatorname{Cov}\left\{{ }^{i} P_{(x)}^{(t)},{ }^{n} P_{(y)}^{(u)}\right\} d y d x \\
& +\int_{r(t)}{ }^{i r} f_{(x)}^{(t)} \int_{b(u)}^{r}{ }^{i a} f_{(y)}^{(u)} \operatorname{Cov}\left\{{ }^{i} P_{(x)}^{(t)},{ }^{i} P_{(y)}^{(u)}\right\} d y d x .
\end{array}\right\}
$$

\subsection{Discounted Financial Projections}

Let $D S(t)$ and $D B(t)$ denote the values of $S(t), B(t)$ discounted to valuation date and $T D S(t)$ and $T D B(t)$ denote the integrals of the discounted values over $(0, t)$. Then, 
Social Security Pension Scheme: An Analytical Approach

$$
\left.\begin{array}{c}
D S(t)=S(t)\left({ }^{\delta} I_{0}^{t}\right)^{-1} \\
D B(t)=B(t)\left({ }^{\delta} I_{0}^{t}\right)^{-1} \cdot
\end{array}\right\}
$$

$S(t)$ depends on the mortality and new entrant factors, which are assumed to be independent of the interest factor. It follows, from the lognormal distribution properties (Appendix B) and [31] of Appendix C, that

$$
\left.\begin{array}{l}
E(D S(t))=E(S(t))\left({ }^{\delta} e_{0}^{t}\right)^{-1}\left({ }^{\delta} v_{0,0}^{t, t}\right)^{1 / 2} \\
V(D S(t))=\left(\begin{array}{c}
V(S(t))\left({ }^{\delta} v_{0,0}^{t, t}\right) \\
+(E(S(t)))^{2}\left(\left({ }^{\delta} v_{0,0}^{t, t}\right)-1\right)
\end{array}\right)\left({ }^{\delta} e_{0}^{t}\right)^{-2}\left({ }^{\delta} v_{0,0}^{t, t}\right) .
\end{array}\right\}^{(*)}
$$

The covariance between $D S(t), D S(t)$, using [35] of Appendix C, is,

$$
\left.\begin{array}{rl}
\operatorname{Cov}( & D S(t), D S(u)) \\
= & \operatorname{Cov}(S(t), S(u))\left({ }^{\delta} v_{0,0}^{t, u}\right)\left({ }^{\delta} e_{0}^{t}\right)^{-1}\left({ }^{\delta} e_{0}^{u}\right)^{-1}\left({ }^{\delta} v_{0,0}^{t, t}\right){ }^{1 / 2}\left({ }^{\delta} v_{0,0}^{u, u}\right)^{1 / 2} \\
& +E(S(t)) E(S(u))\left(\left({ }^{\delta} v_{0,0}^{t, u}\right)-1\right)\left({ }^{\delta} e_{0}^{t}\right)^{-1}\left({ }^{\delta} e_{0}^{u}\right)^{-1}\left({ }^{\delta} v_{0,0}^{t, t}\right)^{1 / 2}\left({ }^{\delta} v_{0,0}^{u, u}\right)^{1 / 2} \cdot
\end{array}\right\}^{(*)}
$$

Similar expressions can be derived for $\operatorname{Cov}(D B(t), D B(u)), \operatorname{Cov}(D B(t), D S(u))$.

Next, consider the total discounts over $(0, t)$, i.e. $T D S(t)$,

$$
\begin{aligned}
T D S(t) & =\int_{0}^{t} D S(z) d z \\
E(T D S(t)) & =\int_{0}^{t} E(D S(z)) d z \\
\operatorname{Cov}(D S(t), T D S(t)) & =\int_{0}^{t} \operatorname{Cov}(D S(z), D S(t)) d z \\
V(T D S(t)) & =\int_{0}^{t} \int_{0}^{t} \operatorname{Cov}(D S(z), D S(w)) d w d z \\
\operatorname{Cov}(T D S(T), T D S(u)) & =\int_{0}^{t} \int_{0}^{u} \operatorname{Cov}(D S(z), D S(w)) d w d z
\end{aligned}
$$

Expressions for similar variances and covariances concerning $D B(z), T D B(z)$ and for covariances involving both $D S(z)$ and $D B(z)$ can be derived on similar lines.

\subsection{The Capitalised Value of Pension Awards}

Let $B c(t)$ denote the value of the pensions awarded at time $t$, discounted 
to the valuation date. The number of awardees are $P_{(r)}^{(t)}$ and the number who will continue to be on this pension roll at time $t+z$ would be $P_{(r+z)}^{(t+z)}$. Let the corresponding unit pension amount, ignoring salary escalation, be denoted by ${ }^{r} f_{(r+z)}^{(t+z)}$. Note that ${ }^{r} f_{(r+z)}^{(t+z)}={ }^{r} f_{(r)}^{(t)}$. For initial population cohorts $(t<r-b)$,

$$
{ }^{i r} f_{(r)}^{(t)}=s a(r-t, 0) \frac{s(r)}{s(r-t)} \operatorname{pr}(r-t)
$$

For new entrant cohorts $(t>r-b)$

$$
{ }^{n r} f_{(r)}^{(t)}=s a(b, 0) \frac{s(r)}{s(b)} \operatorname{pr}(b)
$$

The capitalised value of the award, and its expectation and variance, are given by the following expressions:

$$
\begin{aligned}
B c(t) & ={ }^{r} f_{(r)}^{(t)} \int_{0}^{\omega-r} P_{(r+z)}^{(t+z)}\left({ }^{\delta} I_{0}^{t+z}\right)^{-1} d z \\
E(B c(t)) & ={ }^{r} f_{(r)}^{(t)} \int_{0}^{(\omega-r} E\left\{P_{(r+z)}^{(t+z)}\left({ }^{\delta} I_{0}^{t+z}\right)^{-1}\right\} d z \\
V(B c(t)) & =\left({ }^{r} f_{(r)}^{(t)}\right)^{2} \int_{0}^{\varpi-r} \int_{0}^{\varpi-r} \operatorname{Cov}\left\{P_{(r+z)}^{(t+z)}\left({ }^{\delta} I_{0}^{t+z}\right)^{-1}, P_{(r+w)}^{(t+w)}\left(I_{0}^{t+w}\right)^{-1}\right\} d w d z .
\end{aligned}
$$

Noting that $P_{(r+z)}^{(t+z)},{ }^{\delta} I_{0}^{t+z}$ are independent, the integrand in $E(B c(t))$ could be expressed as,

$$
E\left(P_{(r+z)}^{(t+z)}\right)\left({ }^{\delta} e_{0}^{t+z}\right)^{-1}\left({ }^{\delta} v_{0,0}^{t+z, t+z}\right)^{1 / 2}
$$

Using [35] of Appendix C, the integrand in $V(B c(t))$ could be expressed as,

$$
\left.\begin{array}{c}
\left(\operatorname{Cov}\left(P_{(r+z)}^{(t+z)}, P_{(r+w)}^{(t+w)}\right)\left({ }^{\delta} v_{0,0}^{t+z, t+w}\right)\right)\left({ }^{\delta} e_{0}^{t+z}\right)^{-1}\left({ }^{\delta} v_{0,0}^{t+z, t+z}\right)^{1 / 2}\left({ }^{\delta} e_{0}^{t+w}\right)^{-1}\left({ }^{\delta} v_{0,0}^{t+w, t+w}\right)^{1 / 2} \\
+E\left(P_{(r+z)}^{(t+z)}\right) E\left(P_{(r+w)}^{(t+w)}\right)\left(\left({ }^{\delta} v_{0,0}^{t+z+t+w}\right)-1\right)\left({ }^{\delta} e_{0}^{t+z}\right)^{-1}\left({ }^{\delta} v_{0,0}^{t+z, t+z}\right)^{1 / 2}\left({ }^{\delta} e_{0}^{t+w}\right)^{-1}\left({ }^{\delta} v_{0,0}^{t+w, t+w}\right)^{1 / 2}
\end{array}\right\} .
$$

The covariance between the capitalised value of the awards at time $t$ and the corresponding discounted salary bill will be given by, 


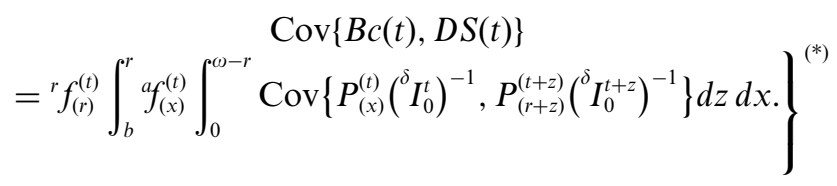

The integrand in (21) can be developed as follows:

$$
\left.\begin{array}{c}
\left(\operatorname{Cov}\left(P_{(x)}^{(t)}, P_{(r+z)}^{(t+z)}\right)\left({ }^{\delta} v_{0,0}^{t, t+z}\right)\right)\left({ }^{\delta} e_{0}^{t}\right)^{-1}\left({ }^{\delta} e_{0}^{t+z}\right)^{-1}\left({ }^{\delta} v_{0,0}^{t, t}\right)^{1 / 2}\left({ }^{\delta} v_{0,0}^{t+z, t+z}\right)^{1 / 2} \\
E\left(P_{(x)}^{(t)}\right) E\left(P_{(r+z)}^{(t+z)}\right)\left(\left({ }^{\delta} v_{0,0}^{t, t+z}\right)-1\right)\left({ }^{\delta} e_{0}^{t}\right)^{-1}\left({ }^{\delta} e_{0}^{t+z}\right)^{-1}\left({ }^{\delta} v_{0,0}^{t, t}\right)^{1 / 2}\left({ }^{\delta} v_{0,0}^{t+z, t+z}\right)^{1 / 2} \cdot
\end{array}\right\}
$$

\subsection{Statistical Distributions}

From the discussion in Appendix B, it will be seen that $P(x, t)$ is lognormally distributed if the new entrant growth and the survival processes are regarded as fractional stochastic processes, which is a reasonable approximation when dealing with populations of the size normally encountered in social insurance pension schemes. As regards the Total Population functions $A(t), R(t)$, they are integrals — which are comparable to sums - of lognormal variates. As explained in Appendix B, although the distribution of the sum of lognormal variates is not known, it has been the practice in various applications to regard the sum of lognormal variates as a lognormal variate. This practice is adopted for the present purposes. A similar consideration applies to the distribution of the functions $S(t), B(t)$.

From (14), in view of the normality assumption of the force of interest, $D S(t), D B(t)$ can be regarded as products of lognormal variables, and therefore as lognormal. $T D S(t), T D B(t)$ are integrals of lognormal variables, and are approximately lognormal, by analogy with the result for the sum of lognormal variables. On similar grounds, $B c(t)$ - see $(18)$ - can also be regarded as being lognormally distributed. Confidence limits can be set for any of these outputs, using Appendix B: [27], [28] or Appendix C: [33], [34].

\section{The Secondary Valuation Outputs}

\subsection{The Demographic Ratio}

The Demographic Ratio expresses the number of pensioners as a proportion of the corresponding number of active persons, that is,

$$
D R(t)=\frac{R(t)}{A(t)}
$$

A classic deterministic valuation will provide only the ratio of the expected values, i.e. 


$$
D R^{D}(t)=\frac{E(R(t))}{E(A(t))} .
$$

On the other hand, a stochastic valuation can provide a confidence interval for this ratio, at any chosen level of significance.

The statistical analysis of ratios is discussed in Appendix $\mathrm{C}$ where it is shown that the analysis is straightforward under the lognormal assumption. It will be seen from Appendix B that the ratio of two lognormal variates is itself a lognormal variate. Let,

$$
\left.\begin{array}{c}
E(R(t))=\mu, E(A(t))=v \\
V(R(t))=\sigma^{2}, V(A(t))=\theta^{2}, \operatorname{Cov}(R(t), A(t))=\phi .
\end{array}\right\}
$$

Since $R(t), A(t)$ are approximately lognormal, it follows from Appendix C, [39] and [40], that the $(100-\varepsilon) \%$ confidence limits for $D R(t)$ are given by,

$$
\left.\begin{array}{c}
\exp \left(a+b \Phi^{-1}(1-\varepsilon / 200)\right) \\
a=\ln \left(\frac{\mu}{v}\left(1+\frac{\theta^{2}}{v^{2}}\right)^{1 / 2}\left(1+\frac{\sigma^{2}}{\mu^{2}}\right)^{-1 / 2}\right) \\
b^{2}=\ln \left(\left(1+\frac{\theta^{2}}{v^{2}}\right)\left(1+\frac{\sigma^{2}}{\mu^{2}}\right)\left(1+\frac{\phi}{\mu \nu}\right)^{-2}\right) .
\end{array}\right\}
$$

where $\Phi^{-1}$ is the inverse function of the distribution function of the standard normal variable.

\subsection{Computation of Premiums under Various Financial Systems}

\subsubsection{General principles}

There is a variety of financial systems applicable to a social insurance pension scheme (Iyer, 1999, chapter 1). The classical method of determining premiums for any given financial system is on the basis of deterministic projections, which is effectively in terms of expected values.

Any financial system essentially partitions the future course of the pension scheme into non-intersecting zones of equilibrium, within each of which a premium is computed as a ratio between a function of the benefit projections (the Numerator, $N$ ) and another function of the salary projections (the Denominator, D). Strictly speaking, the reference here should be to the discounted benefit and salary projections.

The classical method determines the premium as the ratio of the respective expected values, i.e. by the formula,

$$
P R^{D}=\frac{E(N)}{E(D)}
$$


In the stochastic approach, the premium $P R^{S}=N / D$ is treated as the ratio of two variables. It turns out that, in view of the theory developed in this paper, the numerator $N$ and denominator $D$ can be regarded as approximately lognormally distributed, for all the financial systems discussed below. Therefore their ratio can be treated as a lognormal variate. The expected value and variance of $P R^{S}$ can then be computed and confidence limits set for the premium, at any desired level of significance, using the method outlined in Appendix C, [39] and [40] — as in the case of the Demographic Ratio, discussed earlier. Thus the same methodology applies throughout, with the appropriate definitions of the functions $N$ and $D$. Below, for the principal financial systems, the entities required for computing $P R^{D}$ as well as the confidence limits for $P R^{S}$ are indicated, in terms of functions whose expressions have been derived in this paper.

\subsubsection{The Pay-As-You-Go system ( $P A Y G$ )}

In the framework of the continuous formulation of the theory in this paper, the $P A Y G$ system balances the discounted income and outgo at each point in time. Therefore,

$$
\left.\begin{array}{rl}
E(D) & =E(D S(t)) \\
E(N) & =E(D B(t)) \\
V(D) & =V(D S(t)) \\
V(N) & =V(D B(t)) \\
(D, N) & =\operatorname{Cov}(D B(t), D S(t)) .
\end{array}\right\}^{(*)}
$$

\subsubsection{The Terminal Funding system (TF)}

In the framework of the continuous formulation, adopted in this paper, the $T F$ system fully capitalises the pensions awarded at time $t$ by the corresponding premium income. The relevant functions are:

$$
\left.\begin{array}{rl}
E(D) & =E(D S(t)) \\
E(N) & =E(B c(t)) \\
V(D) & =V(D S(t)) \\
V(N) & =V(B c(t)) \\
(D, N) & =\operatorname{Cov}(B c(t), D S(t)) .
\end{array}\right\}
$$

\subsubsection{The Reserve Ratio system ( $R R$ )}

The $R R$ system applies a level premium over an interval of years such that a Fund is accumulated bearing a specified ratio $(\Omega)$ to the level of the expenditure at the end of the period. If a series of successive intervals are 
considered, the relevant functions for the interval $(a, b ; a>0)$ are the following:

$$
\begin{aligned}
E(D)= & E(T D S(b))-E(T D S(a)) \\
E(N)= & E(T D B(b))-E(T D B(a))+\Omega(E(D B(b))-E(D B(a))) \\
V(D)= & V(T D S(b))+V(T D S(a))-2 \operatorname{Cov}(T D S(b), T D S(a)) \\
V(N)= & V(T D B(b))+V(T D B(a))-2 \operatorname{Cov}(T D B(b), T D B(a)) \\
& +\Omega^{2}(V(D B(b))+V(D B(a))-2 \operatorname{Cov}(D B(b), D B(a))) \\
& +2 \Omega(\operatorname{Cov}(D B(b), T D B(b))-\operatorname{Cov}(D B(b), T D B(a))) \\
& -2 \Omega(\operatorname{Cov}(D B(a), T D B(b))-\operatorname{Cov}(D B(a), T D B(a))) \\
\operatorname{Cov}(D, N)= & \operatorname{Cov}(T D B(b), T D S(b))+\operatorname{Cov}(T D B(a), T D S(a)) \\
& -\operatorname{Cov}(T D B(a), T D S(b))-\operatorname{Cov}(T D B(b), T D S(a)) \\
& +\Omega\left(\begin{array}{c}
\operatorname{Cov}(D B(b), T D S(b))-\operatorname{Cov}(D B(b), T D S(a)) \\
-\operatorname{Cov}(D B(a), T D S(b))+\operatorname{Cov}(D B(a), T B S(a))
\end{array}\right) .
\end{aligned}
$$

More generally, $\Omega$ could be varied over time, reducing as the scheme matures. For the first period $(0, b)$, terms involving $(a)$ in (30) would be zero and if there is an initial fund $F(0)$, it would appear with a negative sign in the expression for $E(N)$.

\subsubsection{The General Average Premium system (GAP): average premiums}

The General Average Premium system is based on the concept of a constant premium that will ensure the financial equilibrium throughout the infinite lifetime of the scheme following an actuarial valuation at $t=0$, taking credit for the accumulated fund on valuation date. The GAP can be regarded as the limit, as $n \rightarrow \infty$, of the Average Premium over the interval $(0, n)$, provided this limit exists. The functions for computing this Average Premium are:

$$
\left.\begin{array}{rl}
E(D) & =E(T D S(n)) \\
E(N) & =E(T D B(n))-F(0) \\
V(D) & =V(T D S(n)) \\
V(N) & =V(T D B(n)) \\
(D, N) & =\operatorname{Cov}(T D B(n), \operatorname{TDS}(n)) .
\end{array}\right\}
$$

4.3 The Fund and the Fund Ratio when the Future Contribution Rate Schedule is Given

In certain cases, the future contribution rates would have been already 
fixed, and the actuarial valuation is required to assess the amount of the Fund and/or the Fund Ratio, that is, the ratio of the fund to the benefit expenditure, in future years. Let $F(t)$ denote the Fund, ignoring salary escalation, and $\Omega(t)$ the Fund Ratio. Then,

$$
\Omega(t)=\frac{F(t)}{B(t)}
$$

$F(t)$ can be regarded as a lognormal variate and $\Omega(t)$ as the ratio of lognormal variates and the procedures in Appendices $\mathrm{B}$ and $\mathrm{C}$ applied to estimate the expected value and the confidence limits for either of them. Let $c(t)$ denote the pre-determined contribution rate at time $t$ and $F(0)$ the initial Fund. Let $K(z)=c(z) S(z)-B(z)$. Then, noting that $K(z)$ is independent of ${ }^{\delta} I_{0}^{t}$ and ${ }^{\delta} I_{z}^{t}$,

$$
\left.\begin{array}{c}
F(t)=F(0)\left({ }^{\delta} I_{0}^{t}\right)+\int_{0}^{t} K(z)\left({ }^{\delta} I_{z}^{t}\right) d z \\
E(F(t))=F(0) E\left({ }^{\delta} I_{0}^{t}\right)+\int_{0}^{t} E(K(z)) E\left({ }^{\delta} I_{z}^{t}\right) d z .
\end{array}\right\}
$$

Further, using [35] of Appendix C,

$$
\begin{aligned}
V(F(t))= & F^{2}(0) V\left({ }^{\delta} I_{0}^{t}\right)+2 F(0) \int_{0}^{t} E(K(z)) \operatorname{Cov}\left(\left({ }^{\delta} I_{0}^{t}\right),\left({ }^{\delta} I_{z}^{t}\right)\right) d z \\
& +\int_{0}^{t} \int_{0}^{t} \operatorname{Cov}(K(z), K(w)) \operatorname{Cov}\left(\left({ }^{\delta} I_{z}^{t}\right),\left({ }^{\delta} I_{w}^{t}\right)\right) d w d z \\
& +\int_{0}^{t} \int_{0}^{t} \operatorname{Cov}(K(z), K(w)) E\left({ }^{\delta} I_{z}^{t}\right) E\left({ }^{\delta} I_{w}^{t}\right) d w d z \\
& +\int_{0}^{t} \int_{0}^{t} E(K(z)) E(K(w)) \operatorname{Cov}\left(\left(\left(^{\delta} I_{z}^{t}\right),\left({ }^{\delta} I_{w}^{t}\right)\right) d w d z\right.
\end{aligned}
$$

Furthermore, the covariance between $F(t)$ and $B(t)$ will be given by,

$$
\operatorname{Cov}(F(t), B(t))=\int_{0}^{t} E\left({ }^{\delta} I_{z}^{t}\right) \operatorname{Cov}(B(t), K(z)) d z^{(*)}
$$

Each of the integrands in (34) and (35) can be simplified, by noting that, 


$$
\begin{aligned}
E\left({ }^{\delta} I_{z}^{t}\right)= & \left({ }^{\delta} e_{z}^{t}\right)\left({ }^{\delta} v_{z, z}^{t, t}\right)^{1 / 2} \\
\operatorname{Cov}\left(\left({ }^{\delta} I_{z}^{t}\right),\left({ }^{\delta} I_{w}^{t}\right)\right)= & E\left(\left({ }^{\delta} I_{z}^{t}\right)\left({ }^{\delta} I_{w}^{t}\right)\right)-E\left({ }^{\delta} I_{z}^{t}\right) E\left({ }^{\delta} I_{w}^{t}\right) \\
= & \left(\left({ }^{\delta} v_{z, w}^{t, t}\right)-1\right)\left({ }^{\delta} e_{z}^{t}\right)\left({ }^{\delta} e_{w}^{t}\right)\left({ }^{\delta} v_{z, z}^{t, t}\right)^{1 / 2}\left({ }^{\delta} v_{w, w}^{t, t}\right)^{1 / 2} \\
E(K(z))= & c(z) E(S(z))-E(B(z)) \\
\operatorname{Cov}(K(z), K(w))= & c(z) c(w) \operatorname{Cov}(S(z), S(w))+\operatorname{Cov}(B(z), B(w)) \\
& -c(z) \operatorname{Cov}(B(w), S(z))-c(w) \operatorname{Cov}(B(z), S(w)) \\
\operatorname{Cov}(B(t), K(z))= & c(z) \operatorname{Cov}(B(t), S(z))-\operatorname{Cov}(B(t), B(z)) .
\end{aligned}
$$

\section{Numerical Illustrations}

In order to provide numerical illustrations, it is necessary to give more specific forms to the input functions introduced in the earlier sections.

The following models have been designed so as to meet certain basic conditions. The expected values, variances and covariances of the stochastic factor inputs should take finite values. The variance (for $t>0$ ) should be non-zero and the variance, and preferably the coefficient of variation as well, should increase over time, but at a progressively decreasing rate. This reflects Redington's (Redington, 1952) vision of an "expanding funnel of doubt", with the qualification that, eventually "the process of widening slows down". The covariance should decrease in line with the absolute difference between the reference times or ages. These models, however, are for illustrative purposes only, and are not necessarily intended as a prescription for practical application.

\subsection{New Entrant Growth Factor Inputs}

The force of growth of new entrants, its variance and the associated covariances are modelled as follows ( $T$, to be selected, could be the projection period or even $\infty$ ):

$$
\left.\begin{array}{rl}
E(\rho(t)) & =\rho_{0}+\rho_{1} f(t) \\
V(\rho(t)) & =\left(\rho_{3}(1-f(t))\right)^{2} \\
f(t) & =\frac{\exp \left(-\rho_{2} t\right)-\exp \left(-\rho_{2} T\right)}{1-\exp \left(-\rho_{2} T\right)} \\
\operatorname{Cov}(\rho(t), \rho(u)) & =(V(\rho(t)) V(\rho(u)))^{1 / 2} \exp \left(-k_{\rho}|t-u|\right) .
\end{array}\right\}
$$

The condition $\rho_{2}>0$ is imposed, in order to yield finite values. $E(\rho(t))$ varies smoothly from $\rho_{0}+\rho_{1}$ at the outset to the value of $\rho_{0}$ at time $T$. The 
sign of $E(\rho(t))$ determines whether the number of new entrants is, on average, growing or declining over time, An increasing $\rho(t)$ is modelled by the condition $\rho_{1}<0$, and vice versa.

The condition $\rho_{3}>0$ ensures a positive standard deviation. The variance increases smoothly from zero at the outset to the value $\left(\rho_{3}\right)^{2}$ at time $T$, while the coefficient of variation of $\rho(t)$ increases smoothly from zero at the outset to the value $\rho_{3} / \rho_{0}$ at time $T$, provided $\rho_{0} \neq 0$. If $\rho_{0}=0$, implying an eventually stabilising population, the coefficient of variation is ultimately infinite; if $\rho_{1}=\rho_{0}=0$, this implies a population tending to stabilise from the outset, and the coefficient of variation is infinite from the outset. However, in either case, the variance is finite and has an increasing trend over time. The constant $k_{\rho}(>0)$ implies that the correlation coefficient between $\rho(t), \rho(u)$ reduces in line with the absolute difference $|t-u|$. The higher the value of $k_{\rho}$, the lower the intra-factor correlation.

\subsection{The Interest (Net of Salary Escalation) Factor Inputs}

Although there are sophisticated models for the interest factor (see, for example, Wilkie, 1986; Parker, 1994), the force of interest is modelled more simply, as below.

$$
\left.\begin{array}{rl}
E(\delta(t)) & =\delta_{0}+\delta_{1} g(t) \\
V(\delta(t)) & =\left(\delta_{3}(1-g(t))\right)^{2} \\
g(t) & =\frac{\exp \left(-\delta_{2} t\right)-\exp \left(-\delta_{2} T\right)}{1-\exp \left(-\delta_{2} T\right)} \\
\operatorname{Cov}(\delta(t), \delta(u)) & =(V(\delta(t)) V(\delta(u)))^{1 / 2} \exp \left(-k_{\delta}|t-u|\right) .
\end{array}\right\}
$$

The characteristics are similar to the case in 5.1 above. If a negative expected force of interest is to be excluded, the conditions $\delta_{0}>0, \delta_{0}+\delta_{1}>0$ could be imposed. However, as the normal distribution is assumed for $\delta(t)$, the probability of a negative force of interest cannot be eliminated. A higher value of $k_{\delta}$ implies lower intra-factor correlation, and vice versa.

\subsection{The Mortality Factor Inputs}

Much research has been devoted over the years to the modelling of the projection of mortality rates and their variances and covariances (see e.g. Tuljapurkar and Boe, 1999; Pitacco, 2004; Booth, 2006; Alho, 2007). A prominent model is the Lee-Carter model (see Lee, 2004), which is being further developed by several authors (e.g. Li et al., 2006; Renshaw and Haberman, 2006). For the purposes of the present paper however, which is only demonstrative, a simpler approach is taken below for the projection of mortality and for the computation of variances and covariances. 


\subsubsection{Projection of the expected force of mortality}

As all the variants considered - see Table 4 - assume mortality decline over time, a projection of the expected mortality has been undertaken. Any mortality function might be chosen for the dynamic modelling of mortality (Pitacco, 2004, p281). Accordingly, it is proposed to select the force of mortality for this purpose and to model it as follows ( $T$, to be selected, could be the projection period or even $\infty$ ):

$$
E\left(\mu^{(1)}(x, t)\right)=\hat{\mu}^{(1)}(x, T)+\left(\hat{\mu}^{(1)}(x, 0)-\hat{\mu}^{(1)}(x, T)\right) \chi(t) .
$$

In (39), the force of mortality experienced at age $x$ and time $t$ is denoted by $\mu^{(1)}(x, t), b \leq x<\omega . \hat{\mu}^{(1)}(x, 0)$ on the valuation date is known and the expected force of mortality in year $T, \hat{\mu}^{(1)}(x, T)$ is assumed. $\chi(t)$ is a function such that $\chi(0)=1$ and $\chi(T)=0$. This formulation is comparable to the original, additive, Lee-Carter model (Lee, 2004) and is similar to the exponential interpolation approach, between the current and the optimal life table - see Pitacco, 2004, p284. ( $T$, to be selected, could be the projection period or even $\infty)$.

$$
\chi(t)=\exp \left(-\lambda_{0} t\right) \frac{\exp \left(-\lambda_{0} t\right)-\exp \left(-\lambda_{0} T\right)}{1-\exp \left(-\lambda_{0} T\right)}
$$

\subsubsection{Modelling the variance and covariance of the force of mortality}

They are modelled as ( $T$, to be selected, could be the projection period or even $\infty$ ):

$$
\begin{aligned}
V\left(\mu^{(1)}(x, t)\right)= & \left(\lambda_{2} E\left(\mu^{(1)}(x, t)\right)(1-h(t))\right)^{2} \\
h(t)= & \exp \left(-\lambda_{1} t\right) \frac{\exp \left(-\lambda_{1} t\right)-\exp \left(-\lambda_{1} T\right)}{1-\exp \left(-\lambda_{1} T\right)} \\
\operatorname{Cov}\left(\mu^{(1)}(x, t), \mu^{(1)}(y, u)\right)= & \left(V\left(\mu^{(1)}(x, t)\right) V\left(\mu^{(1)}(y, u)\right)\right)^{1 / 2} \\
& \times \exp \left(-k_{\mu}^{0}|x-y|-k_{\mu}^{1}|t-u|\right) .
\end{aligned}
$$

The expression for the variance implies that the coefficient of variation increases from zero at the outset to the value $\lambda_{2}$ at time $T$. Since a negative force of mortality is to be excluded, this constant should be subject to a limit. For example, under the assumption of the normal distribution for the force of mortality, the condition $\lambda_{2}<\frac{1}{2}$ will ensure that the probability of a negative force of mortality is always less than $2.5 \%$.

The expression for the covariance implies that the correlation between forces of mortality reduces in line with the absolute differences in the age and in the time of reference. Higher values of $k_{\mu}^{0}, k_{\mu}^{1}$ (both $>0$ ) imply lower intrafactor correlation, and vice versa. 


\subsection{Discrete Approximations for Integrals}

For practical application, the integrals at the various stages of the theoretical development have to be numerically approximated. For simple integrals, one of the following formulae has been used, as convenient ( $m$ and $n$ are integers).

$$
\left.\begin{array}{l}
\int_{m}^{n} f(x) d x=\sum_{x=m}^{n-1} f\left(x+\frac{1}{2}\right) \\
\int_{m}^{n} f(x) d x=\frac{1}{2} f(m)+\sum_{x=m+1}^{n-1} f(x)+\frac{1}{2} f(n) .
\end{array}\right\}
$$

For double integrals, in the present context, it was considered adequate to use one of the following simple formulae, although it is noted that there are more refined methods, such as that based on a Fibonacci lattice - see Sloan and Joe, $1994\left(m, m^{*}, n, n^{*}\right.$ are integers).

$$
\left.\begin{array}{rl}
\int_{m}^{n} \int_{m^{*}}^{n^{*}} f(x, y) d y d x= & \sum_{x=m}^{n-1} \sum_{y=m^{*}}^{n^{*}-1} f\left(x+\frac{1}{2}, y+\frac{1}{2}\right) \\
\int_{m}^{n} \int_{m^{*}}^{n^{*}} f(x, y) d y d x= & \sum_{x=m+1}^{n-1} \sum_{y=m^{*}+1}^{n^{*}-1} f(x, y) \\
& +\frac{1}{2}\left(\sum_{x=m+1}^{n-1}\left(f\left(x, n^{*}\right)+f\left(x, m^{*}\right)\right)\right) \\
& +\frac{1}{2}\left(\sum_{y=m^{*}+1}^{n^{*}-1}(f(n, y)+f(m, y))\right) \\
& +\frac{1}{4}\left(f\left(m, m^{*}\right)+f\left(m, n^{*}\right)+f\left(n, m^{*}\right)+f\left(n, n^{*}\right)\right) .
\end{array}\right\}
$$

\subsection{Outline of Model Scheme and Input Data for the Illustrations}

A retirement pension scheme, with the pension formula of 1 per cent of the final salary per year of service, is illustrated. For simplicity, only one sex (males) is considered. Members enter at age 20 and retire at age 65. The pension, after award, is fully adjusted to the level of insured salaries. The scheme is projected from its outset, and pre-scheme service is excluded. Thus there are no initial pensioners and no initial Fund. The starting active population, initial salaries and the assumed salary scale are shown in Table 2.

The methodology follows the lines described in Section 2.2. The basic 
Table 2. Starting population data and salary scale assumption

$\begin{array}{cccccc}\text { Age-groups } & \begin{array}{c}\text { Active } \\ \text { members }\end{array} & \begin{array}{c}\text { Average } \\ \text { salary }(\$)\end{array} & \begin{array}{c}\text { Annual salary } \\ \text { bill }(\text { (\$million) }\end{array} & \text { Age } & \text { Salary scale } \\ 20-25 & 144,079 & 4,901 & 706 & 20 & 100 \\ 25-30 & 136,392 & 7,145 & 974 & 25 & 165 \\ 30-35 & 128,853 & 9,037 & 1,164 & 30 & 221 \\ 35-40 & 121,319 & 10,540 & 1,279 & 35 & 267 \\ 40-45 & 113,579 & 11,672 & 1,326 & 40 & 302 \\ 45-50 & 105,316 & 12,452 & 1,311 & 45 & 328 \\ 50-55 & 96,064 & 12,863 & 1,236 & 50 & 344 \\ 55-60 & 84,827 & 12,976 & 1,101 & 55 & 350 \\ 60-65 & 69,571 & 12,976 & 903 & 60 & 350 \\ & & & & 65 & 350 \\ \text { Total } & 1,000,000 & 10,000 & 10,000 & & \end{array}$

Table 3. Service table and life table for retirees

Service table (active lives)

$\begin{array}{crrrrr}\text { Age } & \text { Initial } & \text { Eventual } & \text { Age } & \text { Initial } & \text { Eventual } \\ 20 & 1,000 & 1,000 & 65 & 1,000 & 1,000 \\ 25 & 996 & 999 & 70 & 842 & 947 \\ 30 & 990 & 996 & 75 & 626 & 861 \\ 35 & 981 & 992 & 80 & 387 & 733 \\ 40 & 969 & 987 & 85 & 179 & 555 \\ 45 & 950 & 978 & 90 & 53 & 344 \\ 50 & 920 & 961 & 95 & 8 & 138 \\ 55 & 871 & 935 & 100 & 0 & 0 \\ 60 & 788 & 881 & & & \\ 65 & 609 & 731 & & & \end{array}$

mortality tables, initial and eventual, have been selected from an international model (United Nations, 1982: Far Eastern (male) pattern), corresponding to life expectancies at birth of 70 and 82 years. The active survival table allows for a disability decrement, but the disability incidence rates are assumed deterministic and time-invariant. These rates have an increasing trend with age, and amount to an average incidence rate of 4 per 1000 over the whole active age range. The mortality rate of disabled persons is assumed at five times the general population mortality rate at age 20 , this multiple reducing to unity at age 70 ; the active service mortality is determined consequentially. Table 3 summarises the initial service table and life table for retirees, as well as the corresponding (expected) eventual tables.

\subsection{The Variants Selected for Illustration}

Starting from a deterministic variant $\mathrm{A}$, the illustrations cover nine other variants, B to J, each of which is at least partly stochastic. 
A first set of variants, A to D, is intended to demonstrate how the uncertainty of the outputs grows as stochasticity is introduced stage by stage, one factor at a time: Variant B involves stochastic growth, C stochastic growth and stochastic mortality, D stochastic growth, mortality and interest. Thus D constitutes the basic stochastic variant, for which a comprehensive stochastic valuation is demonstrated.

A second set of variants is meant to illustrate, in comparison with variant $\mathrm{D}$, the effect of changes in the stochastic characteristics of each factor input. Variants $\mathrm{E}$ and $\mathrm{F}$ relate to the growth factor and illustrate respectively, the effect of a higher factor variance or a higher intra-factor covariance. Variants $\mathrm{G}$ and $\mathrm{H}$ provide a similar illustration for the mortality factor and I and $\mathbf{J}$ for the interest factor.

The standard assumptions are that over the projection period, the expected force of new entrant growth reduces from $1 \%$ to $0 \%$ and the expected mortality falls as shown in Table 3 while the expected force of interest remains constant at 3\%. The details concerning the parameters for each variant are shown in Table 4. These assumptions have been selected for illustrative purposes only, and are not meant to represent the current or projected characteristics of the factors in any particular country or region.

Table 4. The variants selected for illustration

\begin{tabular}{|c|c|c|c|c|c|c|c|c|c|c|}
\hline & A & B & $\mathrm{C}$ & $\mathrm{D}$ & $\mathrm{E}$ & $\mathrm{F}$ & G & $\mathrm{H}$ & I & $\mathrm{J}$ \\
\hline \multicolumn{11}{|c|}{ Growth factor } \\
\hline$\rho_{0}$ & 0 & 0 & 0 & 0 & 0 & 0 & 0 & 0 & 0 & 0 \\
\hline$\rho_{1}$ & 0.01 & 0.01 & 0.01 & 0.01 & 0.01 & 0.01 & 0.01 & 0.01 & 0.01 & 0.01 \\
\hline$\rho_{2}$ & 0.02 & 0.02 & 0.02 & 0.02 & 0.02 & 0.02 & 0.02 & 0.02 & 0.02 & 0.02 \\
\hline$\rho_{3}$ & 0 & 0.005 & 0.005 & 0.005 & 0.01 & 0.005 & 0.005 & 0.005 & 0.005 & 0.005 \\
\hline$k_{\rho}$ & 0 & 0.5 & 0.5 & 0.5 & 0.5 & 0.05 & 0.5 & 0.5 & 0.5 & 0.5 \\
\hline
\end{tabular}

Mortality factor

$\begin{array}{cccllllllll}\lambda_{0} & 0.02 & 0.02 & 0.02 & 0.02 & 0.02 & 0.02 & 0.02 & 0.02 & 0.02 & 0.02 \\ \lambda_{1} & 0.02 & 0.02 & 0.02 & 0.02 & 0.02 & 0.02 & 0.02 & 0.02 & 0.02 & 0.02 \\ \lambda_{2} & 0 & 0 & 0.5 & 0.5 & 0.5 & 0.5 & 0.75 & 0.5 & 0.5 & 0.5 \\ k_{\mu}^{0} & 0 & 0 & 0.25 & 0.25 & 0.25 & 0.25 & 0.25 & 0.125 & 0.25 & 0.25 \\ k_{\mu}^{1} & 0 & 0 & 0.25 & 0.25 & 0.25 & 0.25 & 0.25 & 0.125 & 0.25 & 0.25\end{array}$

Interest factor

$\begin{array}{ccccccccccc}\delta_{0} & 0.03 & 0.03 & 0.03 & 0.03 & 0.03 & 0.03 & 0.03 & 0.03 & 0.03 & 0.03 \\ \delta_{1} & 0 & 0 & 0 & 0 & 0 & 0 & 0 & 0 & 0 & 0 \\ \delta_{2} & 0.02 & 0.02 & 0.02 & 0.02 & 0.02 & 0.02 & 0.02 & 0.02 & 0.02 & 0.02 \\ \delta_{3} & 0 & 0 & 0 & 0.015 & 0.015 & 0.015 & 0.015 & 0.015 & 0.03 & 0.015 \\ k_{\delta} & 0 & 0 & 0 & 0.5 & 0.5 & 0.5 & 0.5 & 0.5 & 0.5 & 0.05\end{array}$

Note: In equations (37) to (41), $T$ has been set at 100 


\subsection{Summary Review of the Results}

Projections were carried out for a period of 100 years for each of the variants in Table 4, and the numerical results are given in Appendix D. A summary of the results is provided in this section, together with graphical representations in Figures 1 to 18 . In each graph, the Deterministic Valuation result (Variant A) is included to serve as a basis of reference.

Figures 1 to 6 , based on Tables D1 to D3 of Appendix D, illustrate the comprehensive stochastic valuation of variant D. Figure 1 shows the upper and lower confidence limits (DU, DL) of the projected Active population (AC) and Retired population (RT). Figure 2 indicates the time trend of the confidence limits for the Demographic Ratio. Figures 3 to 5 depict the comparable trends for the PAYG, Reserve Ratio and Terminal Funding System premiums. Figure 6 illustrates the trend of convergence, to the General Average Premium, of the Average Premium over successively lengthened intervals, starting from valuation date. All the confidence limits relate to the 95 per cent level of significance. These figures clearly illustrate how, with the progress of time, the primary and secondary valuation outputs can diverge more and more from the deterministic projection values, and thus highlight the importance of providing a scientific measure of this divergence through a stochastic valuation.

Figures 7 to 18, based on Tables D4 to D7 of Appendix D, demonstrate how the uncertainty of inputs is transmitted to the outputs and how a stochastic valuation result is affected as the stochasticity of the inputs is modified, in stages. This analysis concentrates on two specific secondary outputs: the Demographic Ratio and the Reserve Ratio System Premiums. The former is the ideal indicator of the effect of changes in the demographic assumptions (i.e. the new entrant growth and mortality factors), while the latter includes the effect of changes in the financial assumptions (i.e. the interest factor).

Figures 7 and 8 illustrate the occurrence and widening of the uncertainty of the outputs as stochastic factors are progressively introduced, in the order, new entrant growth, mortality and interest. They show the results for variants $B, C$ and $D$, in relation to the deterministic variant $A$.

Figures 9 to 12 relate to the new entrant growth factor. The effect of increasing the factor variance is shown in Figures 9 and 10, which compare variant $\mathrm{E}$ against variant $\mathrm{D}$. The effect of increasing the intra-factor covariance is demonstrated in Figures 11 and 12, by comparing variant $F$ with variant $D$. Similar results are shown for the mortality factor in Figures 13 to 16 , which compare variants $G$ and $H$ against $D$. The corresponding comparisons for the interest factor (variants $\mathrm{I}, \mathrm{J}$ and $\mathrm{D}$ ) are provided in Figures 17 and 18. In all cases, the deterministic valuation result (Variant A) serves as the reference base. 

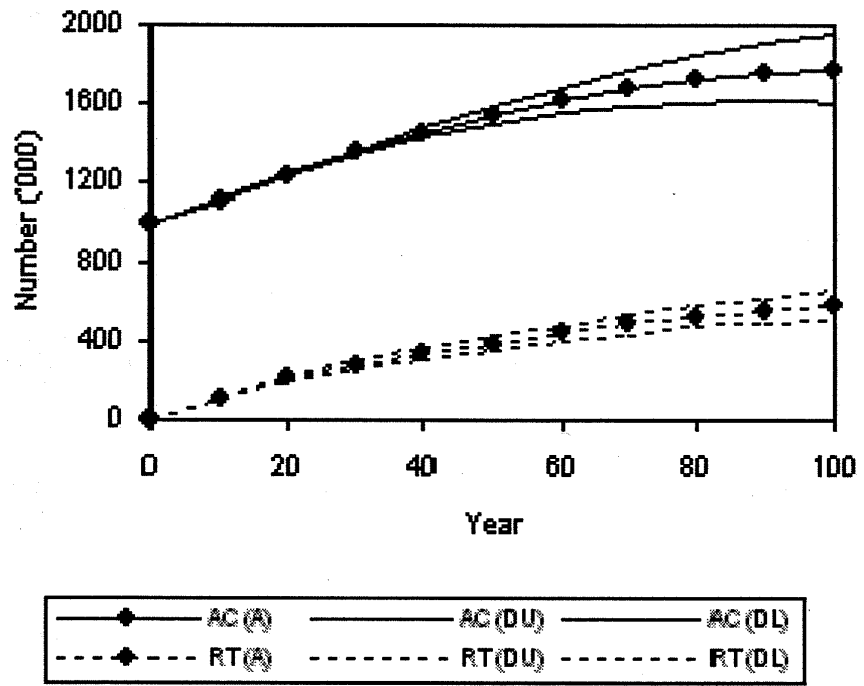

Figure 1. Active persons and retirees (Variant D)
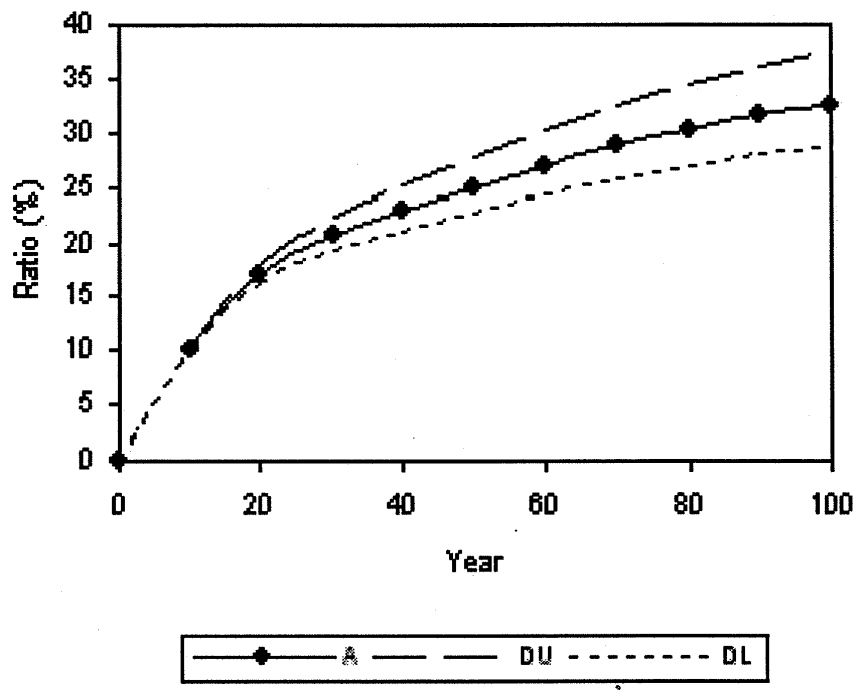

Figure 2. Demographic ratio (Variant D) 

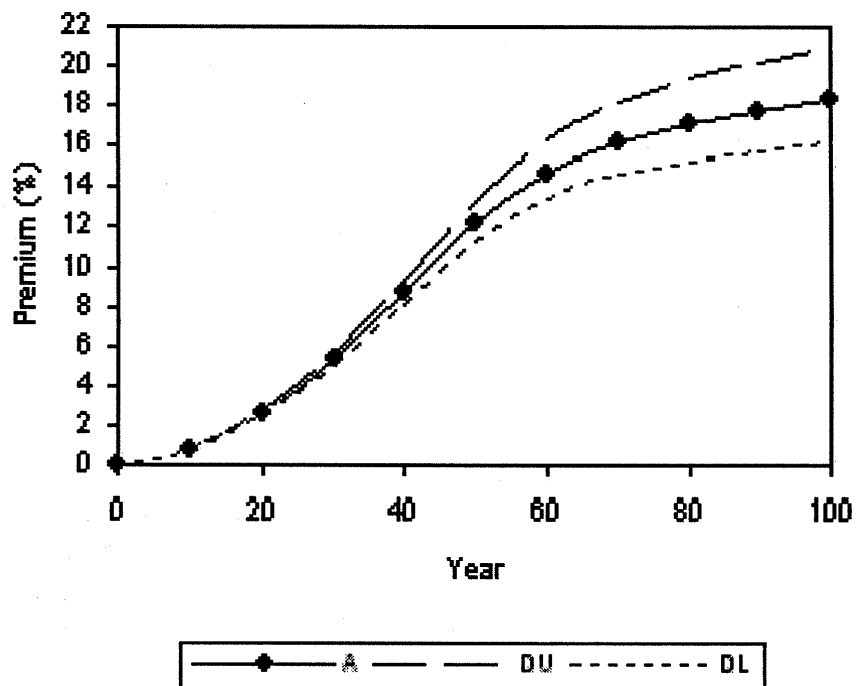

Figure 3. PAYG system (Variant D)
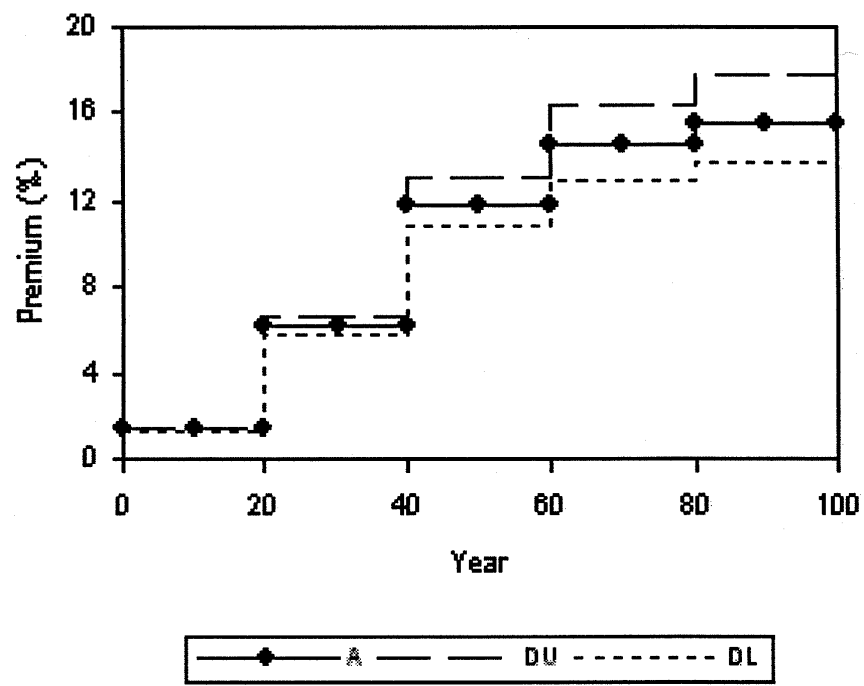

Figure 4. Reserve ratio system (Variant D) 

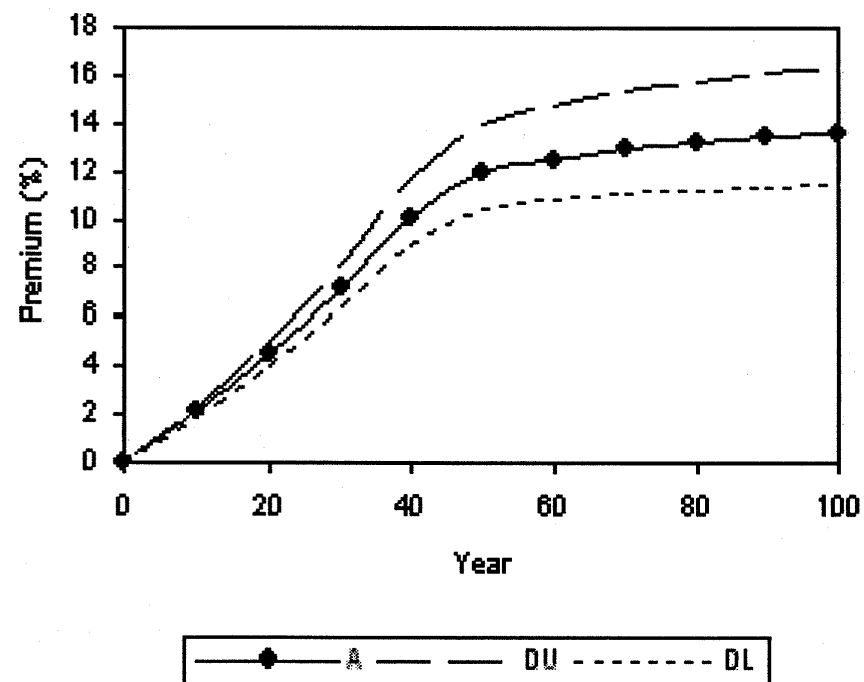

Figure 5. Terminal funding system (Variant D)
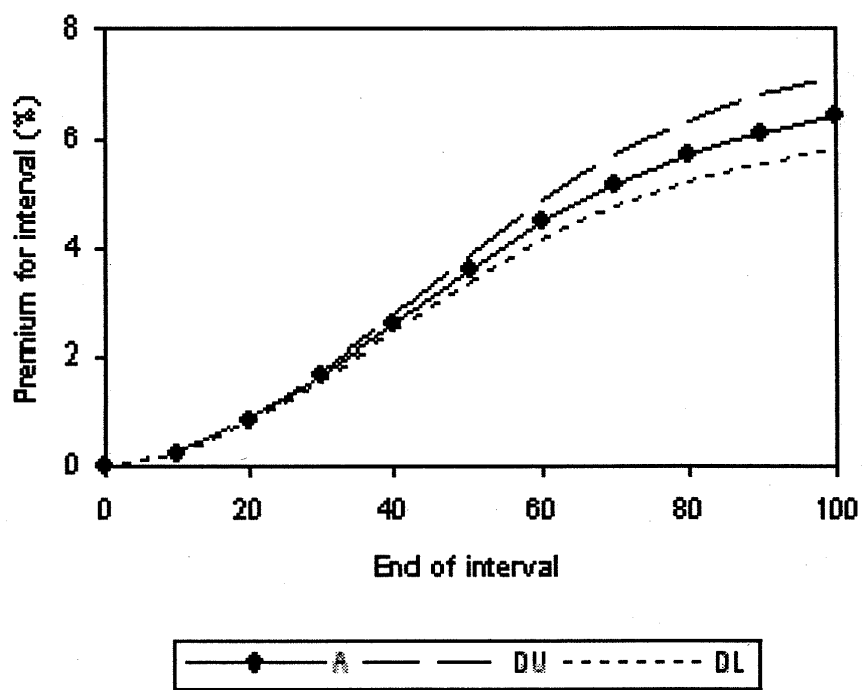

Figure 6. Average premiums (Variant D) 

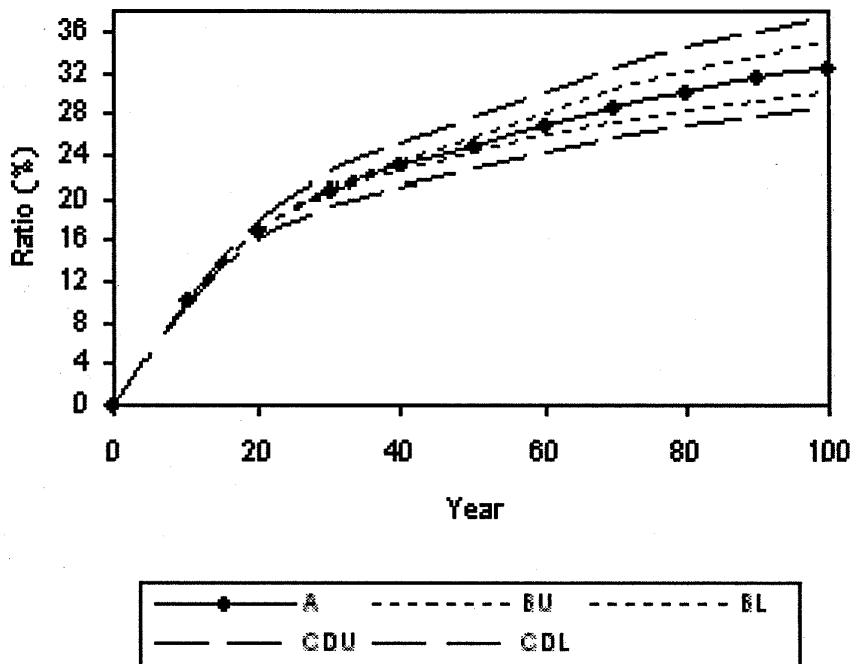

Figure 7. Demographic ratio (Variants B, C and D)
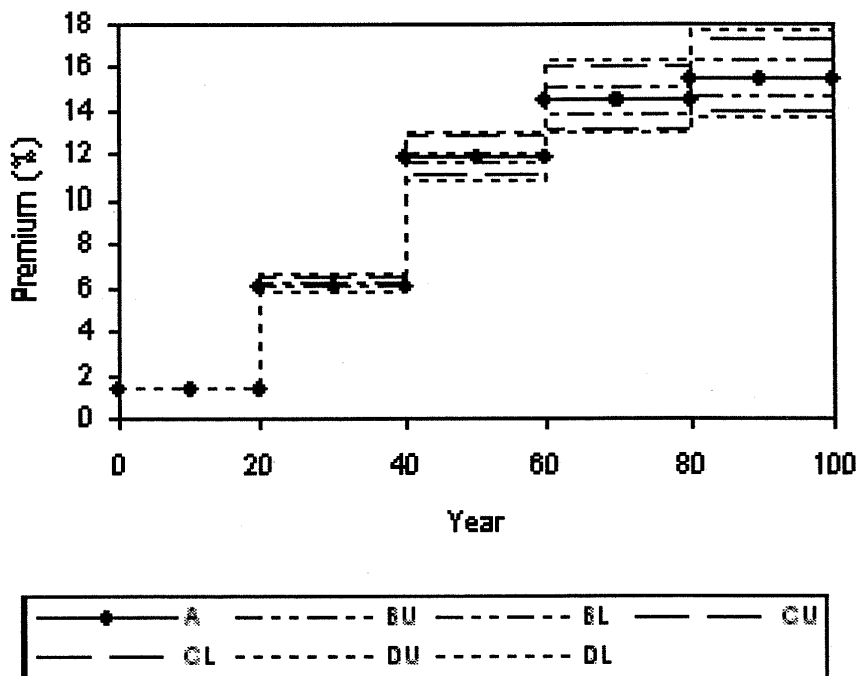

Figure 8. Reserve ratio system (Variants B, C and D) 

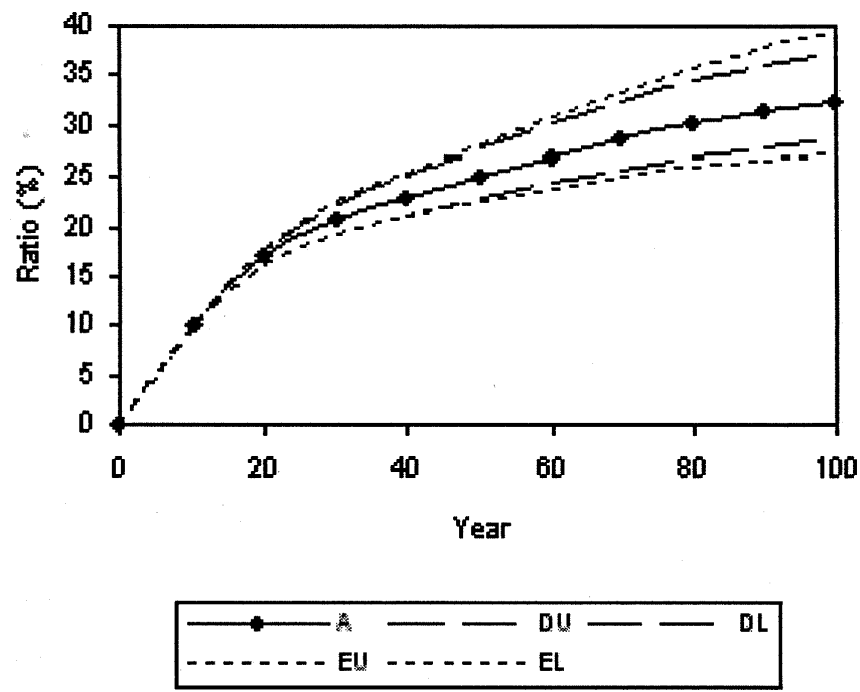

Figure 9. Demographic ratio (Variants D and E)
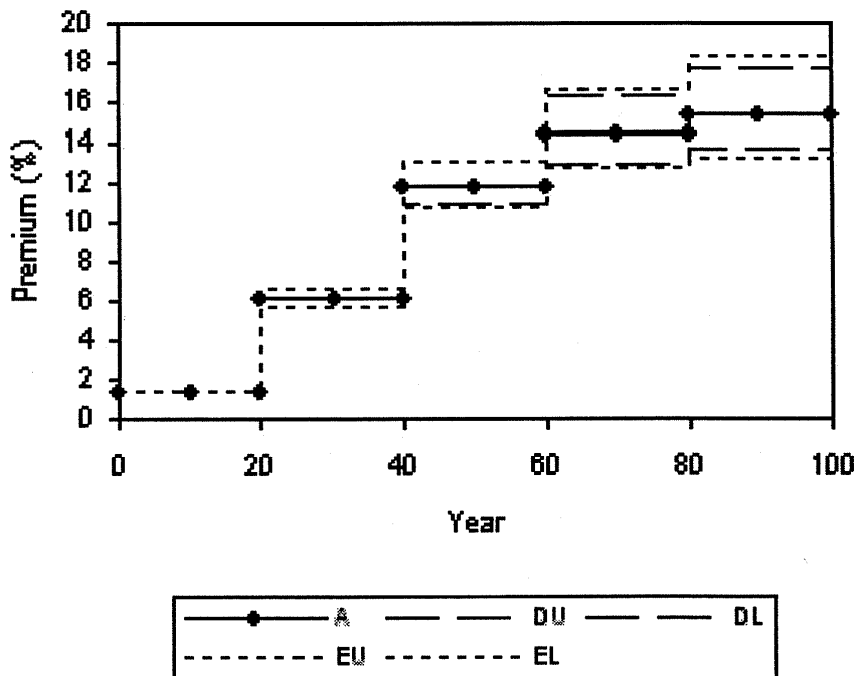

Figure 10. Reserve ratio system (Variants D and E) 

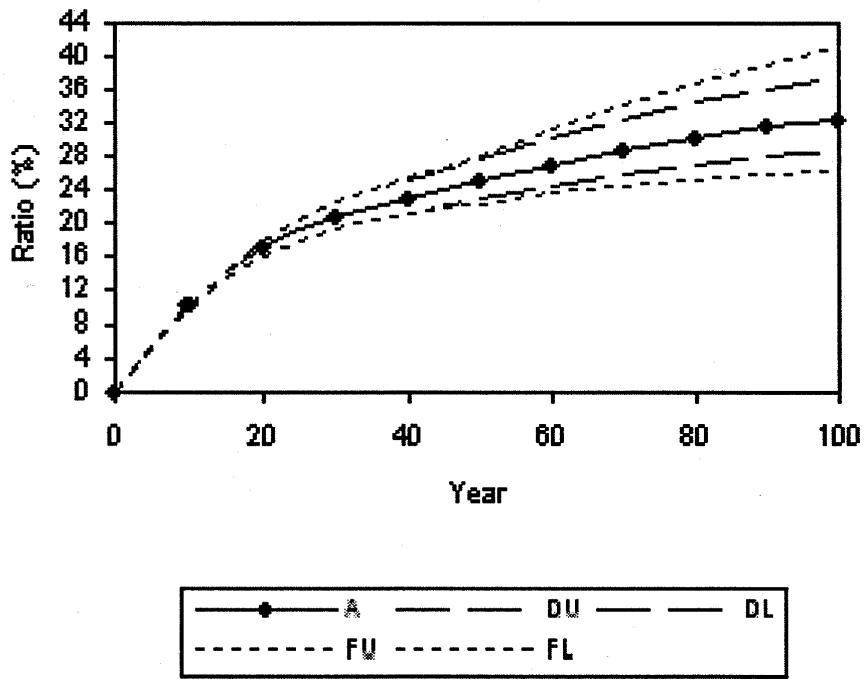

Figure 11. Demographic ratio (Variants D and F)
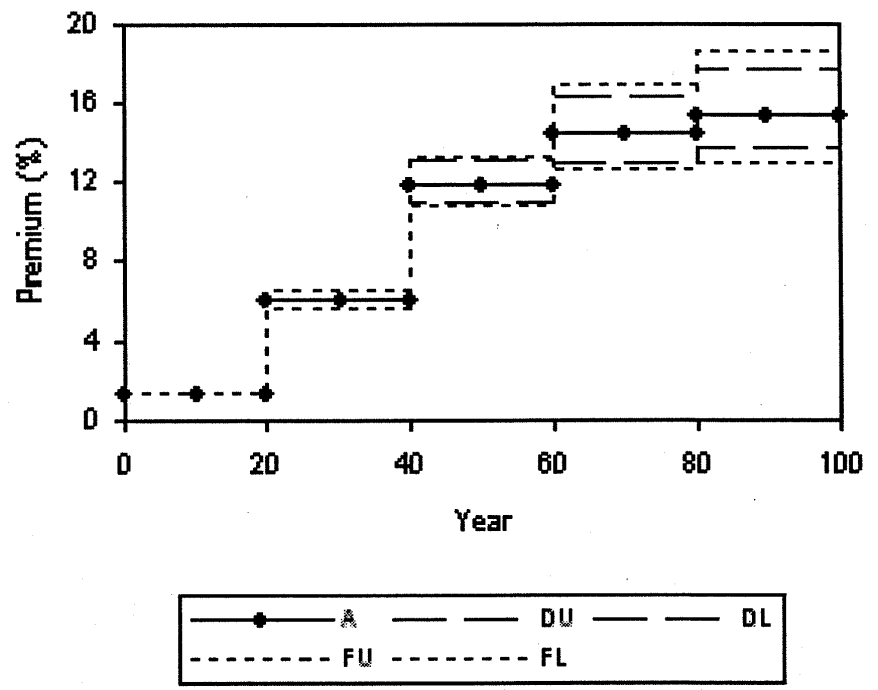

Figure 12. Reserve ratio system (Variants D and F) 

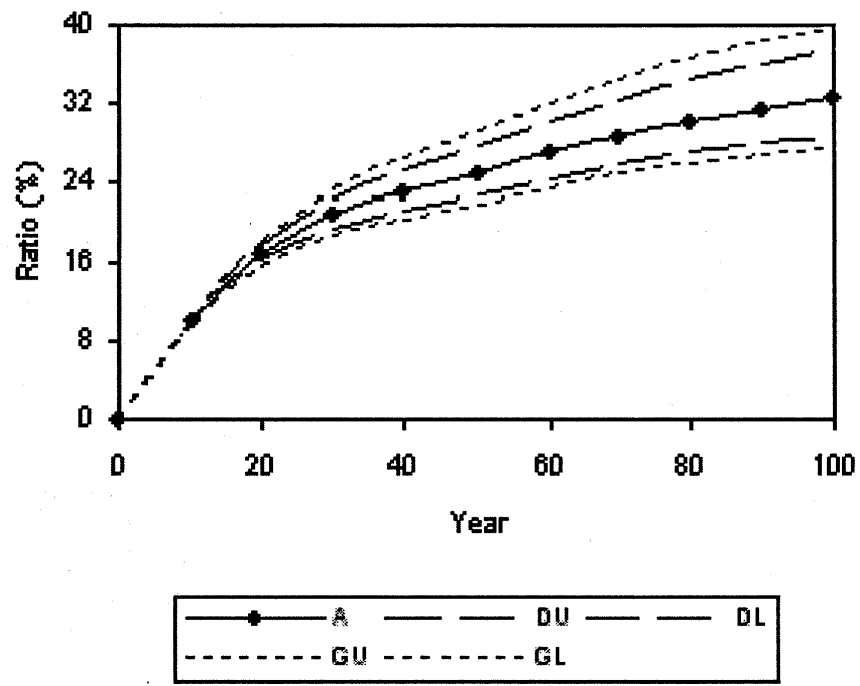

Figure 13. Demographic ratio (Variants D and G)
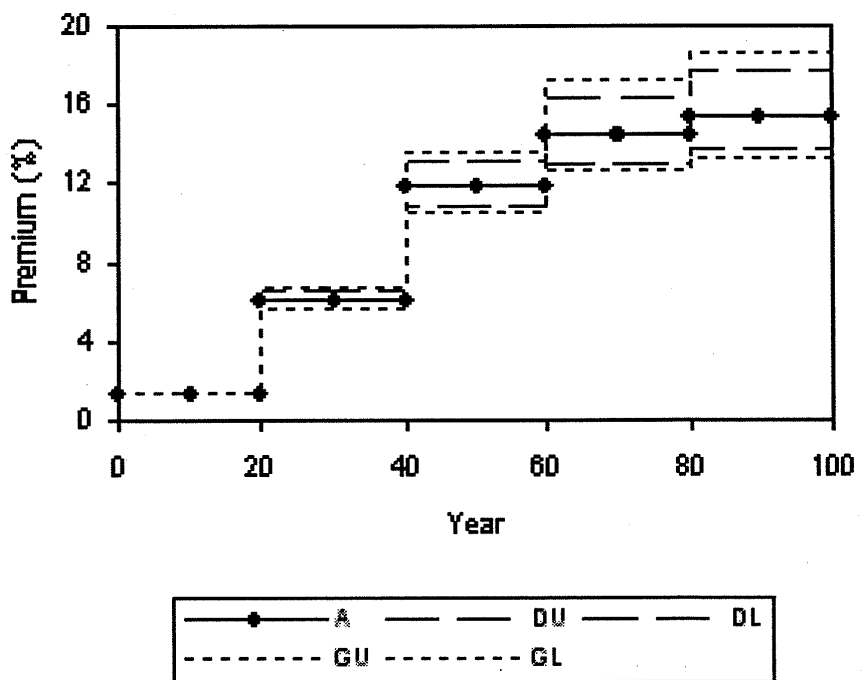

Figure 14. Reserve ratio system (Variants D and G) 

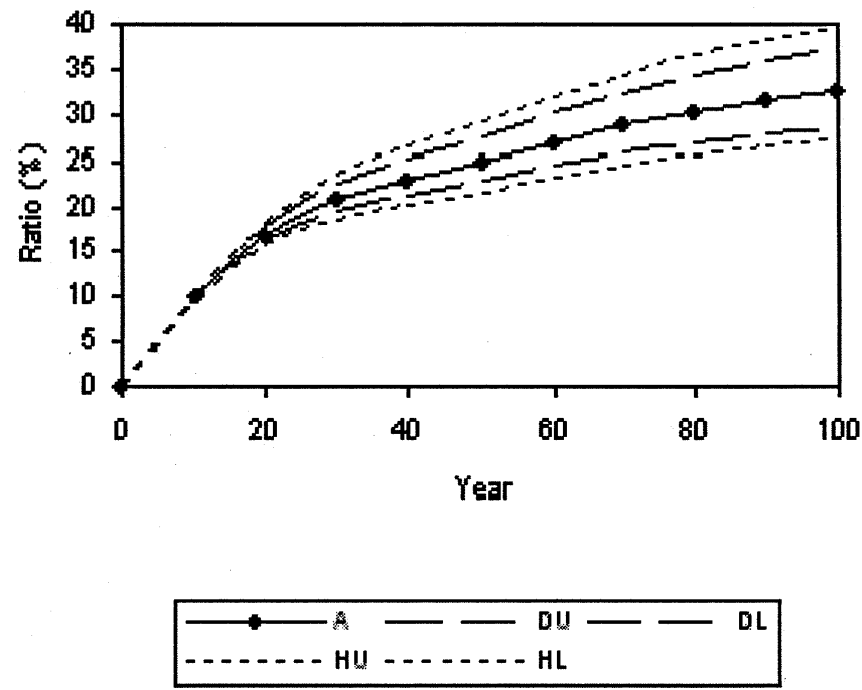

Figure 15. Demographic ratio (Variants D and H)
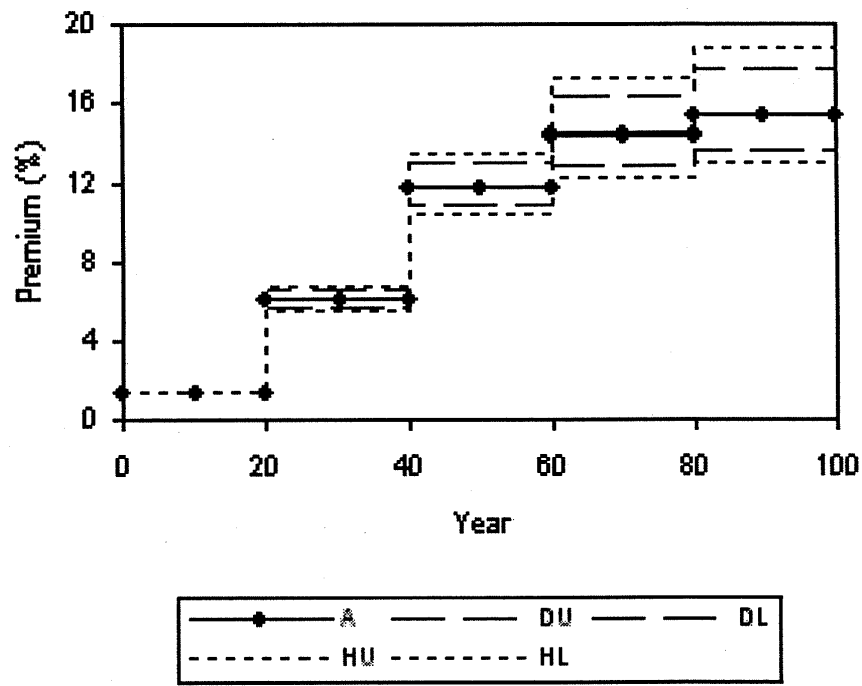

Figure 16. Reserve ratio system (Variants D and H) 

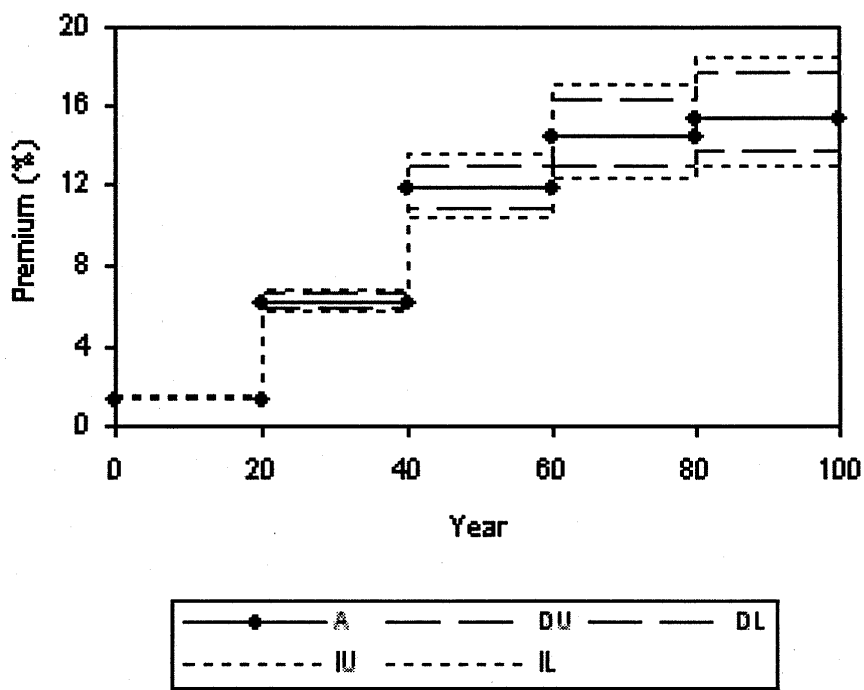

Figure 17. Reserve ratio system (Variants D and I)
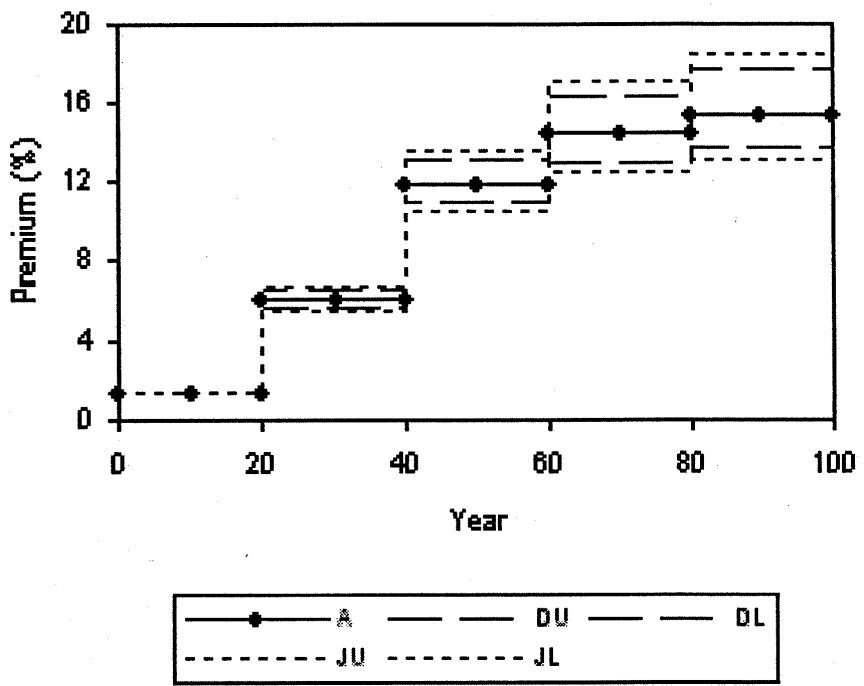

Figure 18. Reserve ratio system (Variants D and J) 


\section{CONCLUSION}

Despite the progress in the application of stochastic methods in other branches of insurance, as well as in related scientific fields such as demography and ecology, recourse to the stochastic approach in social security pension valuations has been very slow to catch on. In fact, to date, there appear to be only two instances, i.e. the United States' OASDI and the Canada Pension Plan, where stochastic methods have been incorporated in the valuation, the former since 2003 and the latter from 2007.

Experts seem to differ widely in their opinion on the complexity, utility and necessity of stochastic valuations in this area. Specific issues are:

- The complexity associated with the difficulty of developing analytical stochastic formulae, due to the number of variables involved and their possible inter-correlations.

- The volume of iteration work involved in applying a fully simulationbased stochastic method.

- The complexity involved in the making of suitable assumptions for the future, in respect of the variances and covariances of the relevant factors, on top of assumptions on their expected values.

- The practicability of determining these assumptions based on the analysis of time series, because of the paucity or unreliability of past data.

- The perception that, unlike other branches of insurance, publicly administered social insurance pension schemes do not operate strictly on generally accepted actuarial principles.

- The impression that the results of stochastic valuations are difficult to communicate to pubic policy makers, who are reputed to prefer, and insist on, a unique "best estimate".

This paper has attempted to address some of the above issues. In particular, it has developed an analytical actuarial model for a social insurance pension scheme. This model demonstrates theoretically the working out of the stochastic processes underlying the evolution of retirement pensions. It treats a range of financial systems applicable to social insurance pension schemes.

Admittedly, the model refers to a simple, retirement pension scheme and considers only three factors as stochastic, which are also assumed to be mutually independent. However, the methodology is quite general, and capable of extension to additional factors and of allowing for inter-factor correlations, although the resulting expressions, inevitably, would be longer and more complex.

The paper has striven to convey the message that a purely deterministic valuation can provide only a very incomplete picture of the evolution of a pension scheme, and could even be misleading. A stochastic valuation is able 
to provide, in addition to the expected values of key outputs - which alone a deterministic valuation can produce - upper and lower limits within which each output could be said to lie, with a specified probability.

The numerical illustrations have demonstrated a comprehensive stochastic valuation and shown how the uncertainty of the valuation results widens as additional input factors are successively taken into account or greater stochasticity is assumed in their regard.

In the author's view, the administering authority of every social insurance pension scheme should consider the possibility of introducing and gradually extending stochastic applications within the framework of actuarial valuations, with the eventual aim of attaining a full-fledged stochastic valuation. In this regard, the model developed in this paper could be a starting point for providing an indication of the stochasticity of retirement pensions, generally the predominant item in a composite pension scheme. Further research is required to extend the model to the other components of the scheme, i.e. disability and survivors' pensions, and to promote and facilitate practical application, with due regard to the proper interpretation and the effective communication of the results of stochastic valuations.

It is fervently hoped that the paper will succeed in drawing attention to this potential, but apparently neglected, area of application of stochastic methods, stimulate interest in further research and eventually, lead to everwidening adoption of stochastic methods in social insurance pension scheme valuations.

\section{REFERENCES}

Alho, J.M. (2007). Methods used in drawing up mortality projections. Fifteenth International Conference of Social Security Actuaries and Statisticians. International Social Security Association, Helsinki.

Alho, J.M. \& Spencer, B.D. (2005). Statistical demography and forecasting, Springer.

AMERICAN ACADEMY OF ACTUARIES (2005). Issue brief: a guide to the use of stochastic models in analyzing social security.

BoARd of Trustees, Federal OASDI Trust Funds (2007). The 2007 annual report of the board of trustees of the federal old-age and survivors insurance and disability insurance trust funds, US Government Printing Office, Washington, D.C.

BongaARts, J. \& Bulatao, R.A. (eds) (2000). Beyond six billion: forecasting the world's population. National Research Council, National Academy Press, Washington, D.C.

Воотн, H. (2006). Demographic forecasting: 1980 to 2005 in review. International Journal of Forecasting, 22(3), 547-581.

Booth, P., Chadburn, R., Cooper, D., Haberman, S. \& James, D. (1999). Modern actuarial theory and practice. Chapman \& Hall/CRC, London.

Buffin, K.G. (2007). Stochastic projection methods for social security systems. PBSS Colloquium, International Actuarial Association, Helsinki.

Burdick, C. \& Manchester, J. (2003). Stochastic models of the social security trust fund. Office of Policy Research, Social Security Administration, Washington, D.C.

Congressional Budget OfFice (2001). Uncertainty in social security's long-term finances: a stochastic analysis. Washington, D.C. 
Daykin, C.D., Pentikainen, T. \& Pesonen, M. (1994). Practical risk theory for actuaries. Chapman \& Hall, London.

DAYKIn, C.D. \& Lewis, D. (1999). A crisis of longer life: reforming pension systems. British Actuarial Journal, 5(1), 55-97.

DAYKIN, C.D. (2000). Social security and the consulting actuary. International Actuarial Association, Social Security Committee.

DAYKIn, C.D. (2001). The role of the Government Actuary's Department in social security in the United Kingdom. British Actuarial Journal, 7(V), 765-790.

Engen, S., Bakke, O. \& Islam, A. (1998). Demographic and environmental stochasticity concepts and definitions. Biometrics, 54, 840-846.

Engen, S. \& SAether, B.-E. (2003). Stochastic population dynamics in ecology and conservation: an introduction. Oxford University Press.

Fenton, L.F. (1960). The sum of lognormal probability distributions in scatter transmission systems. IRE Trans. Commun. Syst., CS(8), 57-67.

FIELleR, E.C. (1932). The distribution of the index in a normal bivariate population. Biometrika, 24, 428-440.

Gillion, C., Turner, J., Bailey, C. \& Latulippe, D. (eds) (2000). Social security pensions: development and reform. International Labour Office, Geneva.

Government Actuary's Department (2003). Government Actuary's Quinquennial Review of the National Insurance Fund as at April 2000. London.

Government Actuary's Department (2005). Variant projections of the update of the Government Actuary's Quinquennial Review of the National Insurance Fund as at April 2000. London.

Hinkley, D.V. (1969, 1970). On the ratio of two correlated random variables. Biometrika, 56(3), 635-639 and 57(3), 683.

Holzmann, R. \& Hinz, R. (2005). Old-age income support in the 21st century. The World Bank, Washington, D.C.

Holzmann, R. \& Palmer, E. (2003). Pension reform: issues and prospects for non-financial defined contribution (NDC) schemes. The World Bank, Washington, D.C.

International Actuarial Association (2003). Final IAA Guidelines of Actuarial Practice for Social Security Programs.

IYER, S. (1999). Actuarial mathematics of social security pensions. International Labour Office, Geneva.

IYER, S. (2003). Application of stochastic methods in the valuation of social security pension schemes. CASS Business School, City University, London.

IYER, S. (2006). A stochastic approach to the actuarial valuation of social security pension schemes. Institute of Insurance and Pension Research, University of Waterloo, Waterloo.

Keilman, N., Pham, D.Q. \& Hetland, A. (2002). Why population forecasts should be probabilistic. Demographic Research, 6(15), 409-454.

Lee, R.D., Anderson, M.W. \& Tuljapurkar, S. (2003). Stochastic forecasts of the social security trust fund. Institute of Business and Economic Research, University of California, Berkeley.

LEE, R. (2004). Quantifying our ignorance: stochastic forecasts of population and public budgets. In Linda I. Waite (ed.). Population and Development Review, V(30), 153-176.

LI, S.-H., Hardy, M.R. \& TAN, K.S. (2006). Uncertainty in mortality forecasting: an extension to the classical Lee-Carter approach. Institute of Insurance and Pension Research, University of Waterloo, Waterloo.

Limpert, E., Stahel, W.A. \& Аввт, M. (2001). Lognormal distributions across the sciences: keys and clues. Bioscience, 51(5), 341-352.

McGillivray, W.R. (1996). Actuarial valuations of social security schemes: Necessity, utility and misconceptions. Social security financing: issues and perspectives. International Social Security Association, Geneva.

Office of the Superintendent of Financial Institutions, Canada: Office of the Chief 
Actuary (2007). Actuarial Report (23rd) on the Canada Pension Plan as at 31 December 2006. Ottawa.

Parker, G. (1994). Two stochastic approaches for discounting actuarial functions. ASTIN Bulletin, 24(2), 167-181.

PiCARD, J.-P. (1996). Valuation of the financial equilibrium of long-term benefit schemes. Social security financing: issues and perspectives. International Social Security Association, Geneva.

Pitacco, E. (2004). Survival models in a dynamic context: a survey. Insurance: Mathematics and Economics, 35, 279-298.

Plamondon, P., Drouin, A., Binet, G., Cichon, M., McGillivray, W.R., Bedard, M. \& Perez-Montas, H. (2002). Actuarial Practice in Social Security. International Labour Office, Geneva.

Redington, F.M. (1952). Review of the principles of life-office valuations. Journal of the Institute of Actuaries, LXXVIII, 286-315.

Regie Des Rentes DU Quebec (2007). Actuarial Report of the Quebec Pension Plan as at 31 December 2006. Quebec.

Renshaw, A.E. \& Haberman, S. (2006). A cohort-based extension to the Lee-Carter model for mortality reduction factors. Insurance: Mathematics and Economics, 38(3), 556-570.

Renshaw, E. (1991). Modelling biological populations in space and time. Cambridge University Press, Cambridge.

SloAN, I.H. \& Joe, S. (1994). Lattice models for multiple integration. Clarendon Press, Oxford.

Social Security Administration (2004). A stochastic model of the long-range financial status of the OASDI program (Actuarial Study No. 117). Office of the Chief Actuary, Washington, D.C.

Social Security Administration (2005). Social Security Programs throughout the World: Africa 2005. Washington, D.C.

Social Security Administration (2006a). Social Security Programs throughout the World: The Americas 2005. Washington, D.C.

Social Security Administration (2006b). Social Security Programs throughout the World: Europe 2006. Washington, D.C.

Social Security Administration (2007). Social Security Programs throughout the World: Asia and the Pacific 2006. Washington, D.C.

Tuljapurkar, S. \& Boe, C. (1998). Mortality change and forecasting: How much and how little do we know? North American Actuarial Journal, 2(4), 13-47.

United Nations (1982). Unabridged model life tables corresponding to the new United Nations Model Life Tables for Developing Countries. New York.

Van Duffel, S., Hoedemakers, T. \& Dhaene, J. (2005). Comparing approximations for risk measures of sums of non-independent lognormal random variables. North American Actuarial Journal, 9(4), 71-82.

Von Luxburg, U. \& Franz, V.H. (2004). Confidence sets for ratios: a purely geometric approach to Fieller's theorem. Max-Planck Institute for Biological Cybernetics, Tubingen.

Wilkie, A.D. (1986). A stochastic investment model for actuarial use. Transactions of the Faculty of Actuaries, 39, 341-403. 
APPENDIX A

\section{BIRTH AND DEATH PROCESSES}

\section{A.1 The Pure Birth Process}

Let $N(t)$ denote a population growing exponentially at the instantaneous rate $\lambda(z)$ (assumed $>0$ ) at time $z$, starting from $N(0)$. Let this process be identified as a " $\lambda$ process". Such a population can be modelled in alternative ways.

\section{A.1.1 $N(t)$ can take fractional values}

Let this process be identified as a "fractional process".

A.1.1.1 Deterministic variant

$N(t)$ is the solution of the differential equation,

$$
\frac{1}{N(t)} \frac{d N(t)}{d t}=\lambda(t)
$$

The solution is given by,

$$
N(t)=N(0) \exp \left\{\int_{0}^{t} \lambda(z) d z\right\} .
$$

This is a purely deterministic result.

\section{A.1.1.2 Stochastic variant}

Suppose $\lambda(t)$ is stochastic and for brevity, let

$$
\alpha(t)=\exp \left\{\int_{0}^{t} \lambda(z) d z\right\}
$$

Note that since $\lambda(t)>0, \alpha(t)>1$.

From [2], it follows that the expected value and variance of $N(t)$ are given by

$$
\left.\begin{array}{l}
E(N(t))=N(0) E(\alpha(t)) \\
V(N(t))=N^{2}(0) V(\alpha(t))
\end{array}\right\} .
$$

It can be shown, on similar lines that,

$$
\operatorname{Cov}\{N(t), N(u)\}=N^{2}(0) \operatorname{Cov}\{\alpha(t), \alpha(u)\} .
$$


A.1.2 $N(t)$ takes integral values only

Let this process be identified as an "integral process".

\section{A.1.2.1 Deterministic variant}

In this case, $N(t)$ has a Negative Binomial distribution (see, e.g. Renshaw, 1991), with expected value and variance given by

$$
\left.\begin{array}{c}
E(N(t))=N(0) \alpha(t) \\
V(N(t))=N(0) \alpha(t)\{\alpha(t)-1\}
\end{array}\right\} .
$$

Thus, even though this variant is deterministic, $N(t)$ is subject to a variance, which arises because of the restriction to integral values. It may be termed the "demographic variance". It can also be shown that,

$$
\operatorname{Cov}\{N(t), N(u), u<t\}=N(0) \alpha(t)\{\alpha(u)-1\} .
$$

\section{A.1.2.2 Stochastic variant}

Suppose $\lambda(t)$ is stochastic. The results in [6] should now be regarded as the conditional expectation and variance of $N(t)$, given $\alpha(t)$. With reference to [2],

$$
E(N(t))=N(0) E(\alpha(t)) .
$$

The variance of $N(t)$ is derived as follows:

$$
\begin{aligned}
V(N(t)) & =E(V(N(t) / \alpha(t)))+V(E(N(t) / \alpha(t))) \\
E(V(N(t) / \alpha(t))) & =N(0)\left\{E\left(\alpha^{2}(t)\right)-E(\alpha(t))\right\} \\
V(E(N(t) / \alpha(t))) & =N^{2}(0) V(\alpha(t)) \\
\therefore V(N(t)) & =N(0)\left\{E\left(\alpha^{2}(t)\right)-E(\alpha(t))\right\}+N^{2}(0) V(\alpha(t)) .
\end{aligned}
$$

It can be shown, on similar lines that,

$\operatorname{Cov}\{N(t), N(u), u<t\}=N(0)\{E(\alpha(t) \alpha(u))-E(\alpha(t))\}+N^{2}(0) \operatorname{Cov}\{\alpha(t), \alpha(u)\}$.

It should be noted that in [9] and [10], the coefficient of $N(0)$ is positive.

\section{A.2 The Pure Death Integral Process}

The pure death process arises when $N(t)$ steadily decreases from the initial value $N(0)$. In this case, $\lambda(z)<0$ so that $0<\alpha(t)<1$. The mathematics is similar to that of the birth process, except that the distribution governing [6] and [7] would be Binomial, and generally lead to identical expressions except in the following cases. 
The expressions in [6] and [7] should now read,

$$
\left.\begin{array}{c}
V(N(t))=N(0) \alpha(t)\{1-\alpha(t)\} \\
\operatorname{Cov}\{N(t), N(u), u<t\}=N(0) \alpha(t)\{1-\alpha(u)\}
\end{array}\right\} .
$$

The expressions in [9] and [10] should read,

$$
\left.\begin{array}{c}
V(N(t))=N(0)\left\{E(\alpha(t))-E\left(\alpha^{2}(t)\right)\right\}+N^{2}(0) V(\alpha(t)) \\
\operatorname{Cov}\{N(t), N(u), u<t\}=N(0)\{E(\alpha(t))-E(\alpha(t) \alpha(u))\}+N^{2}(0) \operatorname{Cov}\{\alpha(t), \alpha(u)\}
\end{array}\right\} .
$$

It should be noted that the coefficient of $N(0)$ in [11] and [12] is positive.

\section{A.3 Generalised Expressions for Integral Processes}

The expressions in [11] and [12] can be expressed more generally to cover both the birth and death processes, using absolute values, as follows.

\section{A.3.1 Deterministic variant}

$$
\left.\begin{array}{c}
V(N(t))=N(0) \alpha(t)\{|1-\alpha(t)|\} \\
\operatorname{Cov}\{N(t), N(u), u<t\}=N(0) \alpha(t)\{|1-\alpha(u)|\}
\end{array}\right\} .
$$

\section{A.3.2 Stochastic variant}

$\left.\begin{array}{c}V(N(t))=N(0)\left\{\left|E(\alpha(t))-E\left(\alpha^{2}(t)\right)\right|\right\}+N^{2}(0) V(\alpha(t)) \\ \operatorname{Cov}\{N(t), N(u), u<t\}=N(0)\{|E(\alpha(t))-E(\alpha(t) \alpha(u))|\}+N^{2}(0) \operatorname{Cov}\{\alpha(t), \alpha(u)\}\end{array}\right\}$.

The expressions in [14] can alternatively be written as,

$$
\begin{aligned}
V(N(t))= & (E(N(t)))\left(\left|1-\frac{E\left(\alpha^{2}(t)\right)}{E(\alpha(t))}\right|\right)+(E(N(t)))^{2} \frac{V(\alpha(t))}{(E \alpha(t))^{2}} \\
\operatorname{Cov}\{N(t), N(u), u<t\}= & (E(N(t)))\left(\left|1-\frac{E(\alpha(t) \alpha(u))}{E(\alpha(t))}\right|\right) \\
& +(E(N(t)))(E(N(u))) \frac{\operatorname{Cov}(\alpha(t), \alpha(u))}{E(\alpha(t)) E(\alpha(u))}
\end{aligned}
$$

\section{A.4 Successive Independent Stochastic Integral Processes}

Suppose starting from $N(0)$, a cohort is subject to a first $\lambda$ process (birth or death) for $t$ years and then to a second, independent $\mu$ process (birth or 
Social Security Pension Scheme: An Analytical Approach

death) for $u$ years. Both processes are assumed to be stochastic integral processes. Let $\lambda(z), \mu(z)$ denote the respective forces of growth and let,

$$
\alpha(t)=\exp \left\{\int_{0}^{t} \lambda(z) d z\right\}, \beta(u)=\exp \left\{\int_{0}^{u} \mu(z) d z\right\} .
$$

Noting that $\beta(u)$ is independent of $\alpha(t)$, the conditional expectation and variance of the result of the second process are,

$$
\begin{gathered}
E(N(t+u) / N(t))=N(t) E(\beta(u)) \\
V(N(t+u) / N(t))=N(t)\left\{\left|E(\beta(u))-E\left(\beta^{2}(u)\right)\right|\right\}+N^{2}(t) V(\beta(u)) .
\end{gathered}
$$

The unconditional expectation will be given by,

$$
E(N(t+u))=E(E(N(t+u) / N(t)))=N(0) E(\alpha(t)) E(\beta(u)) .
$$

Furthermore,

$$
V(N(t+u))=E(V(N(t+u) / N(t)))+V(E(N(t+u) / N(t))) .
$$

Expanding the above relationship and simplifying, the unconditional variance is obtained as,

$$
\begin{aligned}
V(N(t+u))= & N(0)\left\{E(\alpha(t))\left|E(\beta(u))-E\left(\beta^{2}(u)\right)\right|+\left|E(\alpha(t))-E\left(\alpha^{2}(t)\right)\right| E\left(\beta^{2}(u)\right)\right\} \\
& +N^{2}(0)\left\{E\left(\alpha^{2}(t)\right) E\left(\beta^{2}(u)\right)-(E(\alpha(t)) E(\beta(u)))^{2}\right\} \\
= & E(N(t+u))\left(\left|1-\frac{E\left(\beta^{2}(u)\right)}{E(\beta(u))}\right|+\left|1-\frac{E\left(\alpha^{2}(t)\right)}{E(\alpha(t))}\right| \frac{E\left(\beta^{2}(u)\right)}{E(\beta(u))}\right) \\
& +(E(N(t+u)))^{2}\left(\frac{E\left(\alpha^{2}(t)\right)}{(E \alpha(t))^{2}} \frac{E\left(\beta^{2}(u)\right)}{(E \beta(u))^{2}}-1\right) .
\end{aligned}
$$

It can be shown, on similar lines, that the unconditional covariance is given by, 
$\operatorname{Cov}\{N(t+u), N(t+v), v<u\}$

$$
\begin{aligned}
= & N(0)\{E(\alpha(t))|E(\beta(u))-E(\beta(u) \beta(v))| \\
& \left.+\left|E(\alpha(t))-E\left(\alpha^{2}(t)\right)\right| E(\beta(u) \beta(v))\right\} \\
& +N^{2}(0)\left\{E\left(\alpha^{2}(t)\right) E(\beta(u) \beta(v))-(E(\alpha(t)))^{2} E(\beta(u)) E(\beta(v))\right\} \\
= & E(N(t+u))\left(\left|1-\frac{E(\beta(u) \beta(v))}{E(\beta(u))}\right|+\left|1-\frac{E\left(a^{2}(t)\right)}{E(\alpha(t))}\right| \frac{E(\beta(u) \beta(v))}{E(\beta(u))}\right) \\
& +(E(N(t+u)))(E(N(t+v)))\left(\frac{E\left(\alpha^{2}(t)\right)}{(E \alpha(t))^{2}} \frac{E(\beta(u) \beta(v))}{E(\beta(u)) E(\beta(v))}-1\right) .
\end{aligned}
$$

\section{A.5 Parallel Processes}

By "parallel" processes, it is meant that $N(t)$ is generated by a $\lambda$ process starting from $N(0)$ and $M(u)$ is generated by a $\mu$ process starting from $M(0)$, where $\lambda(z)$ and $\mu\left(z^{\prime}\right)$ are correlated. Another case is that of $N(t+v)$ generated by successive independent $\lambda$ and $\mu$ processes and $N(u+w)$ generated by successive independent $\lambda$ and $\gamma$ processes, both starting from $N(0)$, where $\mu(z)$ and $\gamma\left(z^{\prime}\right)$ are correlated. Expressions for the respective expected values and variances follow from results derived above. In addition, defining $\eta(w)$ as:

$$
\eta(w)=\exp \left\{\int_{0}^{w} \gamma(z) d z\right\}
$$

the following expressions can be derived using [35] of Appendix C:

$$
\begin{aligned}
\operatorname{Cov}\{N(t), M(u)\} & =N(0) M(0) \operatorname{Cov}\{\alpha(t), \beta(u)\} \\
& =(E(N(t)))(E(M(u))) \frac{\operatorname{Cov}(\alpha(t), \beta(u))}{E(\alpha(t)) E(\beta(u))}
\end{aligned}
$$

$$
\begin{aligned}
\operatorname{Cov}\{N(t+v), N(u+w), u<t\}= & N(0)[|E(\alpha(t))-E(\alpha(t) \alpha(u))| E(\beta(v) \eta(w))] \\
& +N^{2}(0)[\operatorname{Cov}\{\alpha(t), \alpha(u)\} E(\beta(v) \eta(w)) \\
& +E(\alpha(t)) E(\alpha(u)) \operatorname{Cov}\{\beta(v), \eta(w)\}] \\
= & E(N(t+v))\left(\left|1-\frac{E(\alpha(t) \alpha(u))}{E(\alpha(t))}\right| \frac{E(\beta(v) \eta(w))}{E(\beta(v))}\right) \\
& +(E(N(t+v)))(E(N(u+w))) \\
& \times\left(\frac{E(\alpha(t) \alpha(u))}{E(\alpha(t)) E(\alpha(u))} \frac{E(\beta(v) \eta(w))}{E(\beta(v)) E(\eta(w))}-1\right) .
\end{aligned}
$$


A.6 Remark

It is seen that the variances and covariances for the stochastic integral processes generally consist of two terms. The term containing $N(0)$ is termed the "demographic" variance or covariance, which arises because of the difference between individuals in the outcome at each stage of the process. The term containing $N^{2}(0)$ is termed the "environmental" variance or covariance (see Engen et al., 1998). The latter will generally be the predominant term, and for sufficiently high values of $N(0)$ the first term could be neglected, which facilitates the statistical analysis - see B3 of Appendix B. 


\section{THE LOGNORMAL DISTRIBUTION}

\section{B.1 Introduction}

Like the normal distribution, the lognormal distribution also arises in a surprising variety of branches of science (Limpert et al., 2001) and it has several interesting characteristics.

A random variable $x$ is said to have a lognormal distribution if $z=\ln (x)$ is normally distributed. If the mean and variance of the distribution of $z$ are $\mu_{z}$ and $\sigma_{z}^{2}$, then the distribution of $x$ is said to have parameters $\mu_{z}$ and $\sigma_{z}$, and this is indicated as,

$$
x \rightarrow L N\left[\mu_{z}, \sigma_{z}\right] .
$$

The mean and variance of the above lognormal distribution are given by,

$$
\left.\begin{array}{rl}
\mu_{x} & =\exp \left(\mu_{z}+\frac{1}{2} \sigma_{z}^{2}\right) \\
\sigma_{x}^{2} & =\exp \left(2 \mu_{z}+\sigma_{z}^{2}\right)\left(\exp \left(\sigma_{z}^{2}\right)-1\right) .
\end{array}\right\}
$$

If these relationships are reversed, the following results are obtained:

$$
\left.\begin{array}{l}
\mu_{z}=\ln \left[\frac{\mu_{x}^{2}}{\sqrt{\mu_{x}^{2}+\sigma_{x}^{2}}}\right] \\
\sigma_{z}^{2}=\ln \left[1+\frac{\sigma_{x}^{2}}{\mu_{x}^{2}}\right]
\end{array}\right\}
$$

\section{B.2 Properties of a Pair of Lognormal Variates}

A pair of random variables $x$ and $y$ are said to have a bivariate lognormal distribution if $z=\ln (x)$ and $w=\ln (y)$ have a bivariate normal distribution. Relations similar to [23] and [24] will apply between the means and variances of $y$ and $w$. In addition, the covariances are connected by the relationships,

$$
\left.\begin{array}{l}
\sigma_{x y}=\left(\exp \left(\sigma_{z w}\right)-1\right) \exp \left(\mu_{z}+\frac{1}{2} \sigma_{z}^{2}\right) \exp \left(\mu_{w}+\frac{1}{2} \sigma_{w}^{2}\right) \\
\sigma_{z w}=\ln \left(1+\frac{\sigma_{x y}}{\left|\mu_{x} \mu_{y}\right|}\right) .
\end{array}\right\}
$$


The product and ratio of $x$ and $y$ are also lognormal variates:

$$
\left.\begin{array}{l}
u=x y \rightarrow L N\left[\mu_{z}+\mu_{w}, \sqrt{\sigma_{z}^{2}+\sigma_{w}^{2}+2 \sigma_{z w}}\right] \\
v=\frac{x}{y} \rightarrow L N\left[\mu_{z}-\mu_{w}, \sqrt{\sigma_{z}^{2}+\sigma_{w}^{2}-2 \sigma_{z w}}\right] .
\end{array}\right\}
$$

On the other hand, the distribution of $\tau(=x+y)$ is extremely complex to be treated analytically. However, in several applications, it has been the practice to approximate the distribution of $\tau$ also by a lognormal distribution, although a variety of approaches have been developed for estimating the corresponding parameters $\mu, \sigma$ (see, for example, Fenton, 1960; Vanduffel et al., 2005). If the expectation and variance of $\tau$ are known, the standard approach is to derive these parameters by equating the first two moments. In any case, it can be concluded that the sum of two lognormal variates can be regarded as being approximately lognormal.

The above results concerning the product and sum of two lognormal variates can be generalised to the product or sum of several lognormal variates.

\section{B.3 Statistical Distributions of the Outputs of Stochastic Processes}

In the case of fractional stochastic processes discussed in Appendix A, if it is assumed that the forces of change such as $\lambda(z)$ are normally distributed, it turns out that the results (outputs) of the processes are lognormally distributed. This is because these outputs then assume the exponential form, the exponent being linear combinations (i.e. integrals and their sums) of normal variates. The expected values, variances and covariances of the outputs can then be expressed in terms of the expected values, variances and covariances of the various forces of change, using the properties of the lognormal distribution.

In the case of integral processes, the statistical distribution of the outputs is not so obvious. However, it turns out that, when the size of the population is sufficiently large, such as in the case of social insurance pension schemes, the integral process can be approximated by the corresponding fractional process, and therefore the outputs considered as lognormally distributed.

\section{B.4 Confidence Limits for Lognormal Variates}

If $x \rightarrow L N(a, b)$, since $x$ is a single valued function of the corresponding normal variate, the $(100-\varepsilon) \%$ confidence limits for $x$ would be given by,

$$
\exp \left(a \pm b \Phi^{-1}(1-\varepsilon / 200)\right)
$$


$\Phi^{-1}$ is the inverse function of the distribution function of the standard normal variate and $a$ and $b$ would be given, as in [24], by,

$$
\left.\begin{array}{l}
a=\ln \left[\frac{\mu_{x}^{2}}{\sqrt{\mu_{x}^{2}+\sigma_{x}^{2}}}\right] \\
b^{2}=\ln \left[1+\frac{\sigma_{x}^{2}}{\mu_{x}^{2}}\right] .
\end{array}\right\}
$$

Confidence limits for the product or ratio of lognormal variates are discussed in Appendix C. 
Social Security Pension Scheme: An Analytical Approach

APPENDIX C

\section{STATISTICAL ANALYSIS OF PRODUCTS AND RATIOS}

Let $x$ and $y$ denote stochastic variables with the following main parameters:

$$
\left.\begin{array}{l}
E(x)=\mu ; E(y)=v \\
V(x)=\sigma^{2} ; V(y)=\theta^{2} ; \operatorname{Cov}(x, y)=\phi .
\end{array}\right\}
$$

\section{C.1 Products of Variables}

Consider the variable $w=x y$. The expected value and variance of $w$ can be expressed as follows:

$$
\left.\begin{array}{l}
E(w)=\mu v+\phi \\
V(w)=\sigma^{2} v^{2}+\theta^{2} \mu^{2}+2 \mu \mu_{12}+2 v \mu_{21}+\mu_{22}+2 \mu v \phi-\phi^{2} .
\end{array}\right\}
$$

In the above, $\mu_{r s}$ denotes $E\left((x-\mu)^{r}(y-v)^{s}\right)$. Thus, the variance involves moments of higher order than two, except if $x$ and $y$ are independent, in which case the expressions in [30] would reduce to,

$$
\left.\begin{array}{l}
E(w)=\mu v \\
V(w)=\sigma^{2} v^{2}+\theta^{2} \mu^{2}+\sigma^{2} \theta^{2}
\end{array}\right\}
$$

In the particular case where $x$ and $y$ are lognormally distributed, even if they are correlated, in view of the properties of this distribution (see Appendix B) it can be shown that $w$, which is also lognormal, will have the following expression for the variance, which does not involve the higher order moments:

$$
(\mu \nu+\phi)^{2}\left(\left(1+\frac{\sigma^{2}}{\mu^{2}}\right)\left(1+\frac{\theta^{2}}{v^{2}}\right)\left(1+\frac{\phi}{\mu v}\right)^{2}-1\right) .
$$

Suppose $w \rightarrow L N(a, b)$. Then, with reference to [26] of Appendix B, the $(100-\varepsilon) \%$ confidence limits for $w$ will be given by,

$$
\exp \left(a \pm b \Phi^{-1}(1-\varepsilon / 200)\right)
$$

where $\Phi^{-1}$ is the inverse function of the distribution function of the standard normal variate, and $a$ and $b$ are given by, 


$$
\left.\begin{array}{rl}
a & =\ln \left(\mu \nu\left(1+\frac{\theta^{2}}{v^{2}}\right)^{-1 / 2}\left(1+\frac{\sigma^{2}}{\mu^{2}}\right)^{-1 / 2}\right) \\
b^{2} & =\ln \left(\left(1+\frac{\theta^{2}}{v^{2}}\right)\left(1+\frac{\sigma^{2}}{\mu^{2}}\right)\left(1+\frac{\phi}{\mu \nu}\right)^{2}\right) .
\end{array}\right\}
$$

The above results can be generalised for more than two variables, by including all the possible combinations within each bracket. Two useful results for the mutually independent pairs $(X, Z),(Y, W)$, the variables within each pair being dependent, are

$$
\left.\begin{array}{rl}
\operatorname{Cov}(X, Y Z)= & (E(Y)) \operatorname{Cov}(X, Z) \\
\operatorname{Cov}(X Y, Z W)= & \operatorname{Cov}(X, Z)\{\operatorname{Cov}(Y, W)+(E(Y))(E(W))\} \\
& +(E(X))(E(Z)) \operatorname{Cov}(Y, W) .
\end{array}\right\}
$$

\section{C.2 The Ratio of Two Variables}

Let $z$ denote the ratio of two variables, with parameters as in [29], i.e.

$$
z=\frac{x}{y}
$$

It can be easily shown that, approximately,

$$
\left.\begin{array}{l}
E(z)=\frac{\mu}{v}\left(1+\frac{\theta^{2}}{v^{2}}-\frac{\phi}{\mu v}\right) \\
V(z)=\frac{\mu^{2}}{v^{2}}\left(\frac{\sigma^{2}}{\mu^{2}}+\frac{\theta^{2}}{v^{2}}-2 \frac{\phi}{\mu v}\right) .
\end{array}\right\}
$$

[36] is approximate, since it contains only the first three terms of an infinite expansion, involving higher order terms and moments.

If $x$ and $y$ are normally distributed, the distribution of this quotient $z$ is complex. However, the variable $u$ defined below is approximately distributed as a $\mathrm{N}(0,1)$ variable (Fieller, 1932; Hinkley, 1969, 1970):

$$
u=\frac{v z-\mu}{\left(\sigma^{2} z^{2}-2 \phi z+\theta^{2}\right)} .
$$

This is subject to the condition that the coefficient of variation of $y$, that is, 
$\theta / v$, is small (preferably, less than 3 ), so that the probability of $y$ taking a negative value is negligible. In practice, the above condition may not be satisfied, and confidence limits established for $z$ using the above result might display odd features such as negative values or even open or noncontinuous intervals (see von Luxburg and Franz, 2004).

In case $x$ and $y$ can be assumed to have a Bivariate lognormal distribution, using the properties mentioned in Appendix B, the following exact expressions can be derived:

$$
\left.\begin{array}{l}
E(z)=\frac{\mu}{v}\left(1+\frac{\theta^{2}}{v^{2}}\right)\left(1+\frac{\phi}{\mu v}\right)^{-1} \\
V(z)=(E(z))^{2}\left(\left(1+\frac{\sigma^{2}}{\mu^{2}}\right)\left(1+\frac{\theta^{2}}{v^{2}}\right)\left(1+\frac{\phi}{\mu v}\right)^{-2}-1\right) \cdot
\end{array}\right\}
$$

Also in this case, $z$ is a lognormal variate. Suppose $z \rightarrow L N(a, b)$. Then, with reference to [26] of Appendix B, the $(100-\varepsilon) \%$ confidence limits for $z$ will be given by,

$$
\exp \left(a \pm b \Phi^{-1}(1-\varepsilon / 200)\right)
$$

where $\Phi^{-1}$ is the inverse function of the distribution function of the standard normal variate, and $a$ and $b$ are given by,

$$
\left.\begin{array}{rl}
a & =\ln \left(\frac{\mu}{v}\left(1+\frac{\theta^{2}}{v^{2}}\right)^{1 / 2}\left(1+\frac{\sigma^{2}}{\mu^{2}}\right)^{-1 / 2}\right) \\
b^{2} & =\ln \left(\left(1+\frac{\theta^{2}}{v^{2}}\right)\left(1+\frac{\sigma^{2}}{\mu^{2}}\right)\left(1+\frac{\phi}{\mu v}\right)^{-2}\right) .
\end{array}\right\}
$$


This Appendix presents the numerical results of the illustrations. For the specifications of the variants, reference should be made to Table 4 in the body of the paper. In all tables, DET denotes the Deterministic valuation result and UL and LL denote the upper and lower confidence limits at the $95 \%$ level of significance.

\section{D.1 Stochastic Valuation for Variant D}

The stochastic valuation for variant $\mathrm{D}$ is illustrated in detail in Tables D1 to D3. Table D.1 shows the Demographic projections at 10 year intervals and

Table D.1. Demographic projections and the demographic ratio (Variant D) (equations (11), (12), (24), (25), (26), [27], [28] refer)

Year Active population (1000)

$\begin{array}{rrrr} & \text { DET } & \text { UL } & \text { LL } \\ 0 & 1,000 & 1,000 & 1,000 \\ 10 & 1,114 & 1,116 & 1,112 \\ 20 & 1,233 & 1,238 & 1,227 \\ 30 & 1,347 & 1,361 & 1,333 \\ 40 & 1,452 & 1,479 & 1,426 \\ 50 & 1,542 & 1,587 & 1,499 \\ 60 & 1,616 & 1,684 & 1,550 \\ 70 & 1,674 & 1,770 & 1,584 \\ 80 & 1,718 & 1,843 & 1,602 \\ 90 & 1,750 & 1,906 & 1,608 \\ 100 & 1,772 & 1,958 & 1,604\end{array}$

Retirees (1000)

DET UL LL

$\begin{array}{rr}0 & 0 \\ 113 & 115 \\ 209 & 219 \\ 280 & 302 \\ 333 & 365 \\ 385 & 425 \\ 436 & 484 \\ 481 & 537 \\ 520 & 583 \\ 551 & 622 \\ 575 & 654\end{array}$

Demographic ratio (\%)

DET UL LL

$\begin{array}{rccc}0 & 0 & 0 & 0 \\ 111 & 10.14 & 10.32 & 9.96 \\ 199 & 16.96 & 17.79 & 16.18 \\ 261 & 20.78 & 22.44 & 19.34 \\ 307 & 22.95 & 25.19 & 21.09 \\ 352 & 24.96 & 27.67 & 22.75 \\ 397 & 26.95 & 30.14 & 24.37 \\ 437 & 28.74 & 32.43 & 25.80 \\ 470 & 30.25 & 34.40 & 26.96 \\ 495 & 31.48 & 36.04 & 27.89 \\ 514 & 32.47 & 37.39 & 28.62\end{array}$

Table D.2. Financial projections (ignoring escalation) and PAYG premiums (Variant D) (equations (13), (28), [27], [28], [39], [40] refer)

Year Salary bill (\$ million)

DET UL LL

0
10

20

30

40

50

60

70

80

90

100
10,000

11,161

12,402

13,633

14,783

15,785

16,607

17,261

17,764

18,135

18,391
$10,000 \quad 10,000$

$11,184 \quad 11,138$

$12,457 \quad 12,348$

$13,744 \quad 13,523$

$15,003 \quad 14,569$

$16,177 \quad 15,404$

$17,234 \quad 16,007$

$18,167 \quad 16,406$

$18,975 \quad 16,637$

$19,665 \quad 16,732$

$20,244 \quad 16,717$
Expenditure (\$ million)

DET UL

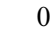

81

326

731

1,278

1,925

2,447

2,801

3,035

3,217

3,360
PAYG premium (\%)

DET UL LL

$\begin{array}{lll}0 & 0 & 0\end{array}$

$\begin{array}{lll}0.72 & 0.73 & 0.71\end{array}$

$\begin{array}{lll}2.62 & 2.72 & 2.53\end{array}$

$\begin{array}{lll}5.35 & 5.65 & 5.08\end{array}$

$\begin{array}{lll}8.64 & 9.27 & 8.09\end{array}$

$\begin{array}{lll}12.19 & 13.26 & 11.28\end{array}$

$\begin{array}{lll}14.73 & 16.30 & 13.43\end{array}$

$\begin{array}{lll}16.22 & 18.23 & 14.60\end{array}$

$\begin{array}{lll}17.08 & 19.38 & 15.26\end{array}$

$\begin{array}{lll}17.74 & 20.24 & 15.75\end{array}$

$\begin{array}{lll}18.26 & 20.95 & 16.14\end{array}$ 
the corresponding demographic ratios. Table D.2 presents the Financial projections and PAYG premiums.

Table D.3 shows the premiums under the Terminal Funding and the

$$
\begin{aligned}
& \text { Table D.3. Funded financial systems (Variant D) } \\
& \text { (equations (29), (30), (31), [39], [40] refer) }
\end{aligned}
$$

Terminal funding premiums

$(\%)$
Average premiums

$(\%)$
Reserve ratio premiums

$(\%)$

Year DET UL LL Interval DET UL LL Interval DET UL LL

\begin{tabular}{rrrrrrrrrrrrr}
0 & \multicolumn{1}{l}{0} & \multicolumn{1}{l}{0} & \multicolumn{1}{l}{0} & & & & & & & \\
10 & 2.10 & 2.29 & 1.94 & $0-10$ & 0.25 & 0.26 & 0.25 & $0-20$ & 1.39 & 1.44 & 1.33 \\
20 & 4.53 & 5.06 & 4.09 & $0-20$ & 0.85 & 0.87 & 0.82 & & & & \\
30 & 7.20 & 8.18 & 6.39 & $0-30$ & 1.66 & 1.74 & 1.59 & $20-40$ & 6.15 & 6.58 & 5.76 \\
40 & 10.11 & 11.65 & 8.88 & $0-40$ & 2.60 & 2.76 & 2.46 & & & & \\
50 & 12.00 & 13.96 & 10.43 & $0-50$ & 3.57 & 3.84 & 3.33 & $40-60$ & 11.85 & 13.03 & 10.85 \\
60 & 12.52 & 14.70 & 10.78 & $0-60$ & 4.45 & 4.85 & 4.12 & & & & \\
70 & 12.92 & 15.30 & 11.04 & $0-70$ & 5.17 & 5.68 & 4.74 & $60-80$ & 14.48 & 16.36 & 12.97 \\
80 & 13.23 & 15.77 & 11.24 & $0-80$ & 5.71 & 6.32 & 5.21 & & & & \\
90 & 13.47 & 16.14 & 11.38 & $0-90$ & 6.12 & 6.81 & 5.55 & $80-100$ & 15.46 & 17.72 & 13.68 \\
100 & 13.65 & 16.42 & 11.50 & $0-100$ & 6.42 & 7.18 & 5.81 & & & &
\end{tabular}

\begin{tabular}{|c|c|c|c|c|c|c|c|}
\hline \multirow[t]{2}{*}{ Variant } & A & \multicolumn{2}{|c|}{$\mathrm{B}^{1}$} & \multicolumn{2}{|c|}{$\mathrm{C}^{2}$} & \multicolumn{2}{|c|}{$\mathrm{D}^{3}$} \\
\hline & DET & UL & LL & UL & LL & UL & LL \\
\hline Year & \multicolumn{7}{|c|}{ Demographic ratio $(\%)$} \\
\hline 0 & 0 & 0 & 0 & 0 & 0 & 0 & 0 \\
\hline 10 & 10.14 & 10.22 & 10.06 & 10.32 & 9.96 & 10.32 & 9.96 \\
\hline 20 & 16.96 & 17.09 & 16.83 & 17.79 & 16.18 & 17.79 & 16.18 \\
\hline 30 & 20.78 & 21.01 & 20.55 & 22.44 & 19.34 & 22.44 & 19.34 \\
\hline 40 & 22.95 & 23.37 & 22.53 & 25.19 & 21.09 & 25.19 & 21.09 \\
\hline 50 & 24.96 & 25.68 & 24.25 & 27.67 & 22.75 & 27.67 & 22.75 \\
\hline 60 & 26.95 & 28.05 & 25.88 & 30.14 & 24.37 & 30.14 & 24.37 \\
\hline 70 & 28.74 & 30.26 & 27.29 & 32.43 & 25.80 & 32.43 & 25.80 \\
\hline 80 & 30.25 & 32.16 & 28.45 & 34.40 & 26.96 & 34.40 & 26.96 \\
\hline 90 & 31.48 & 33.74 & 29.36 & 36.04 & 27.89 & 36.04 & 27.89 \\
\hline 100 & 32.47 & 35.05 & 30.07 & 37.39 & 28.62 & 37.39 & 28.62 \\
\hline
\end{tabular}

Table D.4. Progressive effect of stochasticity

Interval

Reserve ratio system premium (\%)

$\begin{array}{rrrrrrrr}0-20 & 1.39 & 1.39 & 1.37 & 1.42 & 1.35 & 1.44 & 1.33 \\ 20-40 & 6.15 & 6.19 & 6.09 & 6.48 & 5.84 & 6.58 & 5.76 \\ 40-60 & 11.85 & 12.11 & 11.59 & 12.82 & 11.04 & 13.03 & 10.85 \\ 60-80 & 14.48 & 15.06 & 13.90 & 16.07 & 13.21 & 16.36 & 12.97 \\ 80-100 & 15.46 & 16.31 & 14.65 & 17.36 & 13.97 & 17.72 & 13.68\end{array}$

${ }^{1}$ Involves stochastic new entrant growth

${ }^{2}$ Involves stochastic growth and mortality

${ }^{3}$ Involves stochastic growth, mortality and interest 
Reserve Ratio System (for a ratio of 5 and 20 year intervals). It also presents the Average Premiums for intervals starting from valuation date, to illustrate the convergence towards the GAP.

\section{D.2 Sensitivity of a Stochastic Valuation to Changes in Input Characteristics}

Table D.4 indicates the effect of introducing stochasticity in stages, one factor at a time. Table D.5 illustrates the effect of changes in the stochastic characteristics of the growth factor. Tables D.6 and D.7 provide similar information in respect of the mortality and interest factors. In each case, values are shown for two specific outputs, the Demographic Ratio and the Reserve Ratio premium (for a Reserve ratio of 5 and successive intervals of 20 years). DET denotes the Deterministic valuation result (variant A), and UL and LL denote the upper and lower confidence limits at the $95 \%$ level of significance.

Table D.5. Changes in stochastic characteristic of new entrant growth

\begin{tabular}{|c|c|c|c|c|c|c|c|}
\hline \multirow[t]{2}{*}{ Variant } & A & \multicolumn{2}{|c|}{$\mathrm{D}^{1}$} & \multicolumn{2}{|c|}{$\mathrm{E}^{2}$} & \multicolumn{2}{|c|}{$\mathrm{F}^{3}$} \\
\hline & DET & UL & LL & UL & LL & UL & LL \\
\hline Year & \multicolumn{7}{|c|}{ Demographic ratio $(\%)$} \\
\hline 0 & 0 & 0 & 0 & 0 & 0 & 0 & 0 \\
\hline 10 & 10.14 & 10.32 & 9.96 & 10.32 & 9.96 & 10.32 & 9.96 \\
\hline 20 & 16.96 & 17.79 & 16.18 & 17.79 & 16.17 & 17.79 & 16.17 \\
\hline 30 & 20.78 & 22.44 & 19.34 & 22.47 & 19.31 & 22.48 & 19.30 \\
\hline 40 & 22.95 & 25.19 & 21.09 & 25.31 & 20.99 & 25.36 & 20.94 \\
\hline 50 & 24.96 & 27.67 & 22.75 & 27.98 & 22.48 & 28.17 & 22.33 \\
\hline 60 & 26.95 & 30.14 & 24.37 & 30.76 & 23.87 & 31.19 & 23.53 \\
\hline 70 & 28.74 & 32.43 & 25.80 & 33.41 & 25.01 & 34.14 & 24.48 \\
\hline 80 & 30.25 & 34.40 & 26.96 & 35.78 & 25.90 & 36.80 & 25.18 \\
\hline 90 & 31.48 & 36.04 & 27.89 & 37.78 & 26.57 & 39.08 & 25.69 \\
\hline 100 & 32.47 & 37.39 & 28.62 & 39.46 & 27.09 & 40.99 & 26.07 \\
\hline Interval & \multicolumn{7}{|c|}{ Reserve ratio system premium (\%) } \\
\hline $0-20$ & 1.39 & 1.44 & 1.33 & 1.44 & 1.33 & 1.44 & 1.33 \\
\hline $20-40$ & 6.15 & 6.58 & 5.76 & 6.58 & 5.75 & 6.58 & 5.75 \\
\hline $40-60$ & 11.85 & 13.03 & 10.85 & 13.12 & 10.77 & 13.18 & 10.73 \\
\hline $60-80$ & 14.48 & 16.36 & 12.97 & 16.67 & 12.72 & 16.88 & 12.55 \\
\hline $80-100$ & 15.46 & 17.72 & 13.68 & 18.25 & 13.27 & 18.63 & 13.00 \\
\hline
\end{tabular}


Table D.6. Changes in stochastic characteristics of mortality

\begin{tabular}{|c|c|c|c|c|c|c|c|}
\hline \multirow[t]{2}{*}{ Variant } & A & \multicolumn{2}{|c|}{$\mathrm{D}^{1}$} & \multicolumn{2}{|c|}{$\mathrm{G}^{2}$} & \multicolumn{2}{|c|}{$\mathrm{H}^{3}$} \\
\hline & DET & UL & LL & UL & LL & UL & LL \\
\hline Year & \multicolumn{7}{|c|}{ Demographic ratio $(\%)$} \\
\hline 0 & 0 & 0 & 0 & 0 & 0 & 0 & 0 \\
\hline 10 & 10.14 & 10.32 & 9.96 & 10.40 & 9.88 & 10.37 & 9.91 \\
\hline 20 & 16.96 & 17.79 & 16.18 & 18.22 & 15.82 & 18.18 & 15.84 \\
\hline 30 & 20.78 & 22.44 & 19.34 & 23.35 & 18.70 & 23.38 & 18.61 \\
\hline 40 & 22.95 & 25.19 & 21.09 & 26.48 & 20.31 & 26.52 & 20.12 \\
\hline 50 & 24.96 & 27.67 & 22.75 & 29.23 & 21.86 & 29.26 & 21.62 \\
\hline 60 & 26.95 & 30.14 & 24.37 & 31.93 & 23.40 & 31.94 & 23.13 \\
\hline 70 & 28.74 & 32.43 & 25.80 & 34.42 & 24.77 & 34.40 & 24.48 \\
\hline 80 & 30.25 & 34.40 & 26.96 & 36.57 & 25.88 & 36.52 & 25.58 \\
\hline 90 & 31.48 & 36.04 & 27.89 & 38.35 & 26.78 & 38.27 & 26.46 \\
\hline 100 & 32.47 & 37.39 & 28.62 & 39.80 & 27.49 & 39.70 & 27.17 \\
\hline Interval & \multicolumn{7}{|c|}{ Reserve ratio system premium (\%) } \\
\hline $0-20$ & 1.39 & 1.44 & 1.33 & 1.45 & 1.32 & 1.45 & 1.32 \\
\hline $20-40$ & 6.15 & 6.58 & 5.76 & 6.74 & 5.65 & 6.77 & 5.60 \\
\hline $40-60$ & 11.85 & 13.03 & 10.85 & 13.52 & 10.58 & 13.61 & 10.44 \\
\hline $60-80$ & 14.48 & 16.36 & 12.97 & 17.18 & 12.59 & 17.27 & 12.3 \\
\hline $80-100$ & 15.46 & 17.72 & 13.68 & 18.67 & 13.29 & 18.73 & 13.05 \\
\hline
\end{tabular}

Table D.7. Changes in the stochastic characteristics of interest

\begin{tabular}{rrrrrrrr} 
Variant & \multicolumn{1}{c}{$\mathrm{A}$} & \multicolumn{2}{c}{$\mathrm{D}^{1}$} & \multicolumn{2}{c}{$\mathrm{I}^{2}$} \\
& $\mathrm{DET}$ & $\mathrm{UL}$ & $\mathrm{LL}$ & $\mathrm{UL}$ & $\mathrm{LL}$ & $\mathrm{UL}$ & $\mathrm{JL}$ \\
Interval & & \multicolumn{5}{c}{ Reserve ratio system premium (\%) } \\
$0-20$ & 1.39 & 1.44 & 1.33 & 1.47 & 1.30 & 1.46 & 1.31 \\
$20-40$ & 6.15 & 6.58 & 5.76 & 6.78 & 5.58 & 6.77 & 5.59 \\
$40-60$ & 11.85 & 13.03 & 10.85 & 13.52 & 10.45 & 13.50 & 10.46 \\
$60-80$ & 14.48 & 16.36 & 12.97 & 17.03 & 12.44 & 17.01 & 12.46 \\
$80-100$ & 15.46 & 17.72 & 13.68 & 18.54 & 13.05 & 18.50 & 13.09
\end{tabular}

Note: The Demographic ratio is not affected in this case

${ }^{1}$ Basic stochastic variant

${ }^{2}$ Involves a higher factor variance

${ }^{3}$ Involves a higher intra-factor covariance 